\title{
PRÁTICAS DE GESTÃO EM RESTAURANTES PARAENSES MANAGEMENT PRACTICES IN PARÁ RESTAURANTS
}

\author{
Mário César dos Santos de Carvalho ${ }^{1}$ \\ Sérgio Castro Gomes ${ }^{2}$
}

\section{RESUMO}

Competitividade, desempenho e suas implicações para a estratégia das empresas são questões que continuamente despertam o interesse de pesquisadores da área de administração. Uma das formas de analisar como essas organizações combinam seus recursos, com foco em uma gestão mais eficiente e eficaz, se dá por meio das práticas de gestão. O segmento de restaurantes, presente em todas as cidades brasileiras, tem sido tema relevante de pesquisa, principalmente pela área de gestão de turismo, lazer e hospitalidade. Belém do Pará, que recebeu o título de cidade criativa da gastronomia pela UNESCO em 2015, se destaca nesse cenário. O presente artigo buscou, então, identificar os conjuntos de práticas de gestão mais pesquisados na literatura acadêmica recente (2009 a 2018) que possuem aderência ao contexto paraense de gestão de restaurantes. Foram definidas quatro dimensões de práticas: gestão estratégica de recursos humanos, gestão de serviços tecnológicos, gestão da interação humana no encontro de serviços e gestão das práticas de sustentabilidade. Após as entrevistas com os gestores, foram identificadas as práticas de gestão mais frequentes nos restaurantes pesquisados.

Palavras-Chave: Práticas de gestão. Desempenho. Restaurantes.

\section{ABSTRACT}

Competitiveness, performance and its implications for business strategy are issues that continually arouse the interest of business researchers. One way to look at how these organizations combine their resources, focusing on more efficient and effective management, is through management practices.

Manuscript first received/Recebido em 22/10/2018 Manuscript accepted/Aprovado em: 27/06/2019

1 Doutor em Administração pela Universidade da Amazônia - UNAMA e mestre em Administração pela Universidade Federal do Rio Grande do Sul - UFRGS. Diretor Executivo da Ornatos Embalagens e da Filha do Combu Chocolates. Docente da Universidade da Amazônia - UNAMA. E-mail: carvalhomario@yahoo.com.br

2 Doutor em Economia Aplicada pela Universidade Federal de Viçosa. Realiza estágio Pós-Doutoral em Administração de Empresas na Escola de Administração de São Paulo da Fundação Getúlio Vargas - FGV. Pesquisador do Centro de Estudos, Pesquisas e Projetos Estratégicos em Governança Pública da Escola de Governança do Estado do Pará EGPA. Docente no Programa de Pós-Graduação em Administração da Universidade da Amazônia - UNAMA. E-mail: sergio.gomes@unama.br 
The restaurant segment, present in all Brazilian cities, has been a relevant research topic, mainly in the area of tourism, leisure and hospitality management. Belém do Pará, which was awarded the title of creative city of gastronomy by UNESCO in 2015, stands out in this scenario. The present article then sought to identify the most researched sets of management practices in the recent academic literature (from 2009 to 2018) that adhere to the paraense context of restaurant management. Four dimensions of practices were defined: strategic human resources management, technological services management, human interaction management in service encounters and management of sustainability practices. After interviews with managers, the most frequent management practices in the researched restaurants were identified.

Keywords: Management Practices. Performance. Restaurants.

\section{INTRODUÇÃO}

O setor de alimentação fora do lar possui competição intensa, contando com empreendimentos de diversos portes. Nele não há barreiras significativas para novos entrantes, o que leva os restaurantes a reformular estratégias para conquistar e manter clientes. Fatores que outrora garantiam sucesso, tais como: boa localização, ambiente climatizado e atendimento cordial, não são suficientes para lidar com o perfil atual dos consumidores. Na corrida para criar valor e prestar serviços cada vez melhores, é importante que os restaurantes monitorem as percepções dos clientes com relação à qualidade (KEITH; SIMMERS, 2011).

As pesquisas dentro do mainstream da gestão estratégica apresentam aportes teóricos que possibilitam a compreensão dos caminhos que conduzem as empresas a melhores resultados. Entretanto, há espaço para novas perspectivas que aprofundam o conhecimento a respeito da dinâmica de pequenos negócios, envolvendo práticas de gestão menos ortodoxas.

Sem se limitar a discutir estratégias em nível macro, as discussões têm avançado em adaptações estratégicas que iniciam com a criação de uma atmosfera física inovadora (ARIFFIN et al., 2010), passam pela necessidade de aumentar a carga de informações que os clientes atualmente desejam receber sobre a experiência gastronômica (LOUREIRO; RAHMANI, 2016; LEE; SOZEN, 2016; FAKIH et al., 2016; THUNSTRÖM et al., 2016), incluem a percepção do cliente com relação à responsabilidade social corporativa (RHOU et al., 2016; KWOK et al., 2016; LEE et al., 2016b; LINE et al., 2016), e se preocupam com a realidade do lugar onde o restaurante está localizado (BJÖRK; KAUPPINENRÄISÄNEN, 2016; HYUN; PARK, 2016).

Considerando a diversidade de novas práticas de gestão em estudo no setor de restaurantes, o presente artigo se propõe a identificar quais conjuntos de práticas de gestão possuem aderência ao contexto de gestão de restaurantes, mais especificamente na cidade de Belém do Pará.

A capital paraense é referência mundial em gastronomia, recebendo desde 2015 o título internacional de cidade criativa da gastronomia pela UNESCO. Este reconhecimento se deve, entre outros fatores, à originalidade e à fusão de culturas dos pratos regionais. De acordo com dados do Ministério do Turismo, a partir de uma pesquisa realizada em 2016, 99,2\% dos turistas aprovaram (Bom e Muito Bom) suas experiências gastronômicas em Belém, sendo o destino mais bem avaliado do país.

A literatura recente sobre essa temática mostra que existem quatro principais dimensões temáticas, que serão doravante denominadas de dimensões de práticas de gestão. A primeira é a 
vertente de práticas de gestão de recursos humanos (RH) (MURPHY; OLSEN, 2009; AKROUSH et al., 2013; ERHARDT et al., 2016), partindo dos clássicos quatro subsistemas de RH: aquisição, que inclui práticas de planejamento, recrutamento, seleção e descrição de cargos; compensação, que engloba práticas de recompensa monetária e não monetária pelo trabalho realizado; desenvolvimento, que envolve as práticas de avaliação de desempenho, treinamento e desenvolvimento de habilidades e competências; e o subsistema que contempla os aspectos físicos e psicológicos relacionados à saúde e à segurança das pessoas no ambiente de trabalho.

A segunda dimensão se refere às práticas de gestão de serviços na perspectiva tecnológica e está relacionada à crescente utilização de tablets e smartphones nos encontros de serviço (ES), incluindo os momentos que antecedem e os posteriores aos ES, tais como consultas a cardápios, acesso ao sistema de reservas e pedidos on-line (DiPIETRO et al., 2012; ZHENG; GUO, 2016; WEI et al., 2016).

A terceira dimensão se relaciona ao fator humano no ES, especificamente no que tange à percepção de qualidade do cliente frente aos esforços que o staff do restaurante empreende para tornar mais prazerosos os minutos que os consumidores passam no estabelecimento (BARNES et al. 2016; JIN et al., 2016; NIKBIN et al., 2013; LEE et al., 2016a). Essas são práticas expressas pelos comportamentos e expressões corporais, percebidas em termos de inovações, que vão desde a gestão do tempo de espera no restaurante (KREMER; DEBO, 2016), até a capacidade de oferecer um atendimento que surpreenda positivamente os clientes (ABECASSIS-MOEDAS et al., 2016), sempre com foco em detectar e resolver problemas rapidamente.

A quarta dimensão tem relação com as práticas de sustentabilidade que os restaurantes adotam por iniciativa própria, acreditando no valor intrínseco dessas práticas, ou, por opção estratégica, incorporando a utilização de insumos orgânicos na preparação dos pratos, a responsabilidade social com relação à origem dos alimentos, privilegiando os fornecedores locais, além de práticas de prevenção de desperdícios e gestão adequada dos resíduos.

As próximas seções do trabalho estão estruturadas da seguinte forma: os procedimentos metodológicos apresentam as etapas para construção da revisão sistemática de literatura e da pesquisa junto aos gestores dos restaurantes; os resultados descrevem cada uma das dimensões de práticas de gestão analisadas, que se constituíram em categorias na análise de conteúdo, com a apresentação das falas do entrevistados, explicitando as práticas de gestão que possuem aderência ao contexto paraense de gestão de restaurantes; as conclusões apresentam reflexões sobre os conjuntos de práticas de gestão evidenciados na pesquisa, as limitações do estudo, as implicações gerenciais e os caminhos para uma agenda de pesquisa no setor de restaurantes no Brasil.

\section{PROCEDIMENTOS METODOLÓGICOS}

Para operacionalizar a pesquisa foram necessárias duas etapas, descritas nas próximas subseções. A primeira se refere à busca por artigos sobre gestão de restaurantes, com o propósito de analisar os estudos de vanguarda nos periódicos mais relevantes da área de turismo, lazer e hospitalidade. Em seguida, foi realizada a etapa de verificação da aderência desses conjuntos de práticas de gestão ao contexto paraense, com as entrevistas in loco em cinco restaurantes da cidade de Belém do Pará. 


\subsection{REVISÃO SISTEMÁTICA DA LITERATURA}

A primeira etapa metodológica se constituiu em uma revisão sistemática da literatura em periódicos internacionais da área de hospitalidade, buscando identificar os conjuntos de práticas de gestão mais pesquisados acerca do gerenciamento de restaurantes, no período de 2009 a 2018 . Este recorte temporal mais recente se justifica pelo intuito de conhecer a vanguarda dos estudos de práticas de gestão de restaurantes. A coleta de dados teve como ponto de partida o portal Scimago Journal \& Country Rank (SJR). No index de periódicos, utilizou-se o filtro assunto (subject): gestão de turismo, lazer e hospitalidade e foram escolhidos os 20 periódicos mais bem classificados.

Esse ranking fornece, além do indicador SJR, o índice $\mathrm{H}$, que quantifica a produtividade e o impacto de pesquisas individuais ou em grupos baseando-se nos artigos mais citados. Para compor a tabela 1 foi pesquisado também o ranking do fator de impacto do Journal Citation Reports (JCR) da ISI Web of knowledge, medida que reflete o número médio de citações de artigos científicos publicados em determinado periódico. Por último, verificou-se a classificação - quadriênio 2013-2016 - no Qualis da Coordenação de Aperfeiçoamento de Pessoal de Nível Superior (CAPES) e a quantidade de artigos por periódico no assunto e período especificados.

Em seguida, para buscar os artigos, foi aplicado um filtro pela palavra restaurant especificamente no título e nas palavras-chave dos artigos, para evitar que na base houvesse artigos de escopo mais amplo, que utilizavam restaurantes como campo de pesquisa. Entende-se que, quando se coloca a palavra "restaurant" no título e nas keywords do paper, o fenômeno de estudo tem maiores chances de englobar esse tipo de organização.

Tabela 1 - Base de periódicos para análise da literatura sobre de práticas de gestão em restaurantes

\begin{tabular}{c|ccccc}
\hline Periódicos & $\begin{array}{c}\text { Scimago rank } \\
\text { indicator }\end{array}$ & $\begin{array}{c}\mathrm{H} \\
\text { index }\end{array}$ & $\begin{array}{c}\text { Fator de } \\
\text { impacto } \\
\text { JCR }\end{array}$ & $\begin{array}{c}\text { Qualis } \\
\text { CAPES }\end{array}$ & Artigos \\
\hline Academy of Management & 8,55000 & 266 & 7,417 & A1 & 2 \\
Tourism Management & 3,02700 & 143 & 5,921 & A1 & 5 \\
Journal of Travel Research & 2,82000 & 103 & 5,169 & A1 & 1 \\
Annals of Tourism Research & 2,26200 & 132 & 5,086 & A1 & - \\
Sport Management Review & 1,15900 & 39 & 3,516 & - & - \\
Current Issues in Tourism & 1,47400 & 50 & 3,462 & - & 3 \\
International Journal of Hospitality & 2,02700 & 82 & 3,445 & A1 & 102 \\
Management & 1,48800 & 40 & 3,414 & - & 1 \\
Journal of Service Management & 1,54300 & 76 & 3,329 & - & 3 \\
Journal of Sustainable Tourism & 1,31900 & 67 & 3,117 & - & 3 \\
Applied Geography & 1,22000 & 57 & 2,919 & A1 & 4 \\
Journal of Retailing and Consumer Services & 1,45200 & 60 & 2,874 & A1 & 20 \\
International Journal of Contemporary & 1,11000 & 62 & 2,704 & - & - \\
Hospitality Management & 2,15000 & 50 & 2,685 & - & 9 \\
Cities & 1,16000 & 36 & 2,683 & A2 & 38 \\
Journal of Hospitality and Tourism Research & 1,31500 & 37 & 2,449 & - & - \\
Journal of Hospitality Marketing and & & & & & Continua.. \\
Management & & & &
\end{tabular}




\begin{tabular}{c|ccccc}
\hline Continuação Tabela 1 & \multicolumn{1}{c}{} & & \\
\hline Periódicos & $\begin{array}{c}\text { Scimago rank } \\
\text { indicator }\end{array}$ & $\begin{array}{c}\text { H } \\
\text { index }\end{array}$ & $\begin{array}{c}\text { Fator de } \\
\text { impacto } \\
\text { JCR }\end{array}$ & $\begin{array}{c}\text { Qualis } \\
\text { CAPES }\end{array}$ & Artigos \\
\hline Journal of Services Marketing & 1,04000 & 81 & 2,408 & & 16 \\
Leisure Studies & 0,86500 & 52 & 2,247 & A1 & 1 \\
Journal of Vacation Marketing & 0,87500 & 51 & 2,17 & - & 1 \\
Tourism Geographies & 1,20200 & 45 & 2,068 & A1 & - \\
Cornell Hospitality Quarterly & 1,19800 & 58 & 2,06 & - & 64 \\
Journal of Travel and Tourism Marketing & 0,94000 & 52 & 1,975 & A1 & 7 \\
Journal of Hospitality and Tourism & 0,95000 & 21 & 0 & A2 & 8 \\
Management & 0,26000 & 19 & 0 & - & 93 \\
Journal of Foodservice Business Research & TOTAL DE ARTIGOS & & & & $\mathbf{3 8 1}$ \\
\hline
\end{tabular}

Fonte: Elaborado pelos autores, 2019.

Buscava-se estudar restaurantes de serviço tradicional, de pequeno porte, com atendimento realizado à mesa, por garçons. Assim, foram excluídos os estudos feitos em restaurantes de rede e de franquias, assim como os que oferecem apenas o serviço de buffet a quilo. Foram eliminados os artigos que tratavam de questões relacionadas a fast food; questões de branding e de responsabilidade social corporativa; temas de finanças e contabilidade; questões étnicas em relação a desempenho; avaliações de críticos de restaurantes; consumo de vinho; efeitos de crises e catástrofes; e discussões sobre legislação, pois haveria peculiaridades locais enviesando o escopo do artigo.

Após análise dos títulos, resumos e palavras-chave a lista definitiva apresentou 381 artigos. $\mathrm{O}$ agrupamento das práticas de gestão foi feito em quatro dimensões que foram utilizadas para orientar a segunda etapa da pesquisa.

\subsection{PRÁTICAS DE GESTÃO EM RESTAURANTES DE BELÉM}

A pesquisa empírica se baseou em entrevistas semiestruturadas com gestores de cinco restaurantes da cidade de Belém do Pará. Essas entrevistas foram presenciais e aconteceram nos próprios estabelecimentos entre os meses de janeiro e fevereiro de 2017 e cada uma teve duração média de 75 minutos, sendo as falas gravadas e depois transcritas. Em seguida, os dados foram tabulados e analisados por meio da técnica de análise de conteúdo, partindo de categorias pré-definidas.

No quadro 1 os restaurantes pesquisados aparecem codificados e são apresentadas algumas características importantes desses estabelecimentos. É válido frisar que nenhum deles pertence a redes, que suas trajetórias de gestão têm origem em empreendimentos familiares e que nenhum deles foi comprado ou adquirido após o início de suas operações no mercado paraense. 
Quadro 1 - características dos restaurantes pesquisados

\begin{tabular}{|c|c|c|c|c|}
\hline Código & Especialidades & $\begin{array}{c}\text { Anos de } \\
\text { mercado }\end{array}$ & $\begin{array}{c}\text { Capacidade de } \\
\text { atendimento }\end{array}$ & Sistema de serviço \\
\hline A & Churrascaria e cozinha paraense & 45 & 100 lugares & Buffet a quilo e rodízio \\
\hline B & Cozinha paraense & 36 & 200 lugares & À la carte \\
\hline C & Cozinha paraense & 73 & 150 lugares & À la carte e buffet livre \\
\hline D & Cozinha italiana e paraense & 25 & 120 lugares & À la carte \\
\hline E & Cozinha paraense & 12 & 300 lugares & À la carte \\
\hline
\end{tabular}

Fonte: Elaborado pelos autores, 2019.

Todos os estabelecimentos foram escolhidos pelo critério de conveniência e se localizam em Belém-PA. O acesso aos entrevistados foi possível pela rede construída entre os pesquisadores e os restaurantes associados à ABRASEL Pará. Desta forma foi possível saber que os entrevistados exerciam papel de protagonismo nos negócios, atuando do atendimento ao cliente à cozinha, se necessário.

Os restaurantes escolhidos possuem tempo de mercado distintos. Enquanto o mais jovem tinha 12 anos de mercado, o mais longevo passava de 70 anos de funcionamento. Partiu-se do entendimento que isso aumentaria as chances de esses estabelecimentos já terem experimentado uma gama de práticas de gestão que os auxiliaram na superação de situações adversas no dia-a-dia de gestão. A definição do número de casos levou em consideração o critério de saturação, a partir do momento em que os depoimentos dos gestores não traziam novos elementos para análise. O objetivo era apresentar aos entrevistados, durante a leitura das perguntas, os conjuntos de práticas mais investigados pelo universo acadêmico, na vanguarda da pesquisa sobre o tema.

Na seção de resultados são apresentados trechos das entrevistas com o propósito de evidenciar as práticas de gestão levantadas na revisão de literatura que apresentam aderência à realidade brasileira de gestão dos restaurantes. Essas práticas são resultantes de combinações de recursos e de capacidades, que se traduzem em rotinas, relatadas pelos gestores em exemplos de processos formais e informais da dinâmica profissional.

\section{RESULTADOS}

As dimensões de práticas de gestão utilizadas para estruturar as categorias de análise englobam desde aspectos estruturais até o gerenciamento da experiência do cliente nos encontros de serviço. A partir da revisão da literatura, foram consolidadas quatro dimensões de práticas de gestão em restaurantes, as quais estruturam a discussão dos resultados.

Para cada dimensão, os resultados da revisão de literatura e das entrevistas são apresentados de forma integrada. Em cada um dos quadros de análise são apresentadas as categorias de práticas encontradas na literatura especializada, com suas respectivas referências e os principais trechos das entrevistas com os gestores dos cinco restaurantes, que evidenciam a ocorrência dessas práticas e a consequente aderência ao contexto paraense. 


\subsection{RECURSOS HUMANOS}

As práticas de gestão estratégica de recursos humanos $(\mathrm{RH})$ são analisadas na literatura recente como gatilho para melhoria do desempenho nos restaurantes. Estudos que focam em aspectos da qualidade interna de serviços nos restaurantes englobam práticas de recrutamento (maior relevância); retenção; motivação; treinamento; comunicação interna; e segurança no emprego (AKROUSH et al., 2013).

Sistemas de gestão de alto desempenho também envolvem práticas de compensação monetária ou não monetária, advindas de avaliações de desempenho; participação em resultados; promoções internas; empowerment; autonomia de equipes; relações mais horizontais; bons desenhos de cargos; mensuração das práticas de RH; qualidade de vida e de trabalho; diversidade; e orgulho de trabalhar na empresa (MURPHY; OLSEN, 2009).

Essas práticas de gestão de recursos humanos podem ser agrupadas em quatro subsistemas (IVANCEVICH, 2008): aquisição, que envolve o planejamento, a análise e descrição de cargos, os processos de recrutamento e de seleção de pessoas; compensação, que envolve aspectos de remuneração monetária, não-monetária e benefícios; treinamento e desenvolvimento; e saúde e segurança, física e psicológica dos recursos humanos.

A partir dessas práticas, o conteúdo das entrevistas foi analisado em busca de evidências que explicitassem a presença das práticas nas rotinas organizacionais. Com relação ao planejamento de demanda e capacidades dos recursos humanos, todos os gestores afirmaram possuir equipes com as qualificações requeridas para exercerem suas funções, especificamente nas práticas de recrutamento e seleção, com os respectivos reflexos no planejamento da demanda de RH.

Entretanto, devido à dificuldade de encontrar profissionais prontos, com as qualificações desejadas, na maioria dos casos os empresários entrevistados preferem formar seus profissionais. Com isso, eles também encontram soluções interessantes para dois outros problemas inerentes às rotinas de aquisição de RH: turnover e sucessão para cargos que requerem capacidades mais avançadas.

As principais práticas de gestão relacionadas à aquisição de RH estão listadas no quadro 2. Percebe-se que as práticas de contratação são conservadoras, privilegiando indicações pessoais, o que, na visão dos gestores, gera comprometimento de quem indica e de quem é indicado. Critérios rígidos de contratação, com preferência por recrutamento interno, evidenciam a busca por baixos índices de rotatividade e mostra a maturidade com que os gestores buscam conduzir seus negócios, pois compreendem os custos envolvidos com desligamento, contratação e treinamento de novos funcionários. Além disso, o turnover gera impacto no nível de serviço, o que deve ser evitado no segmento de restaurantes. 


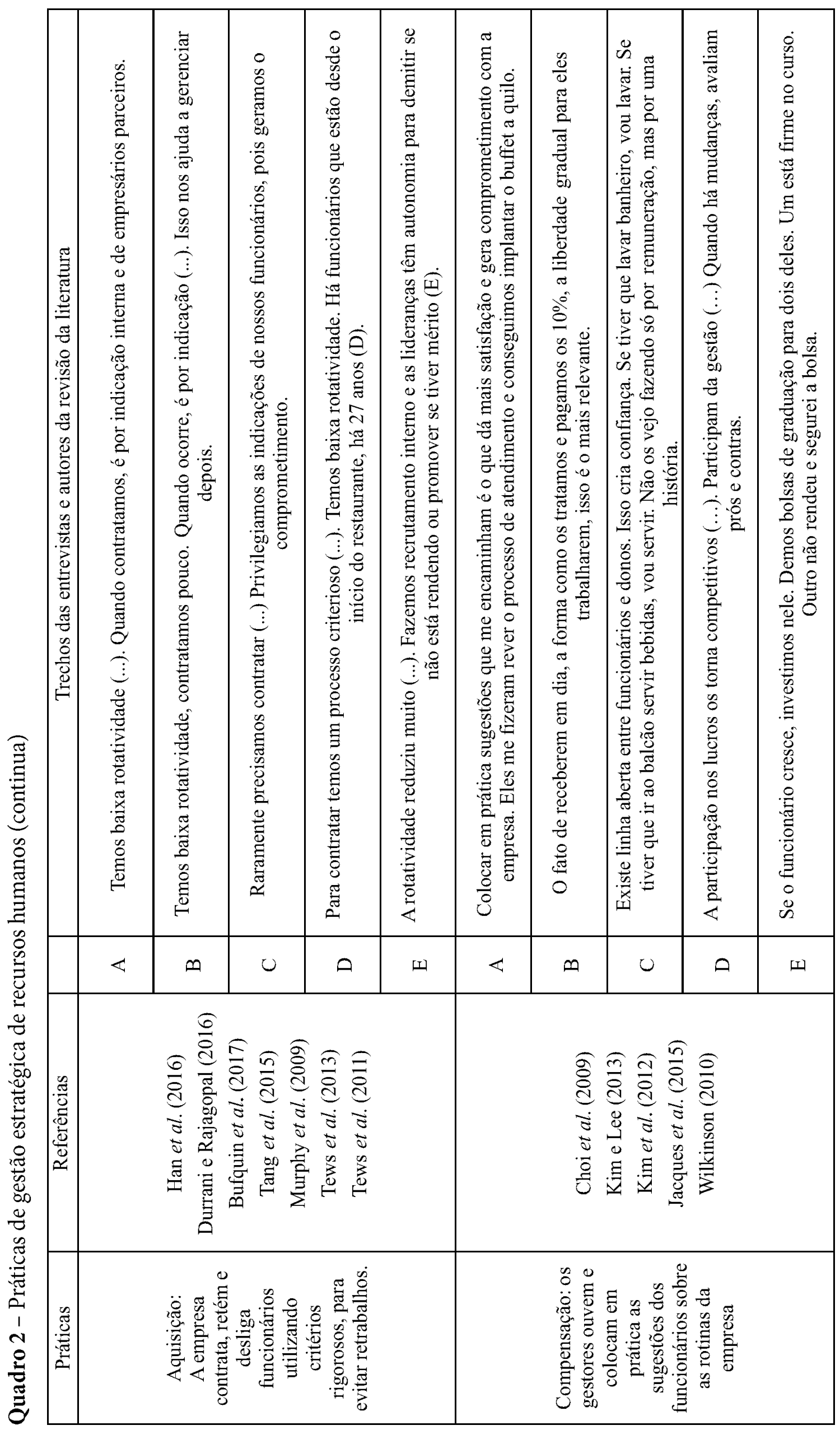




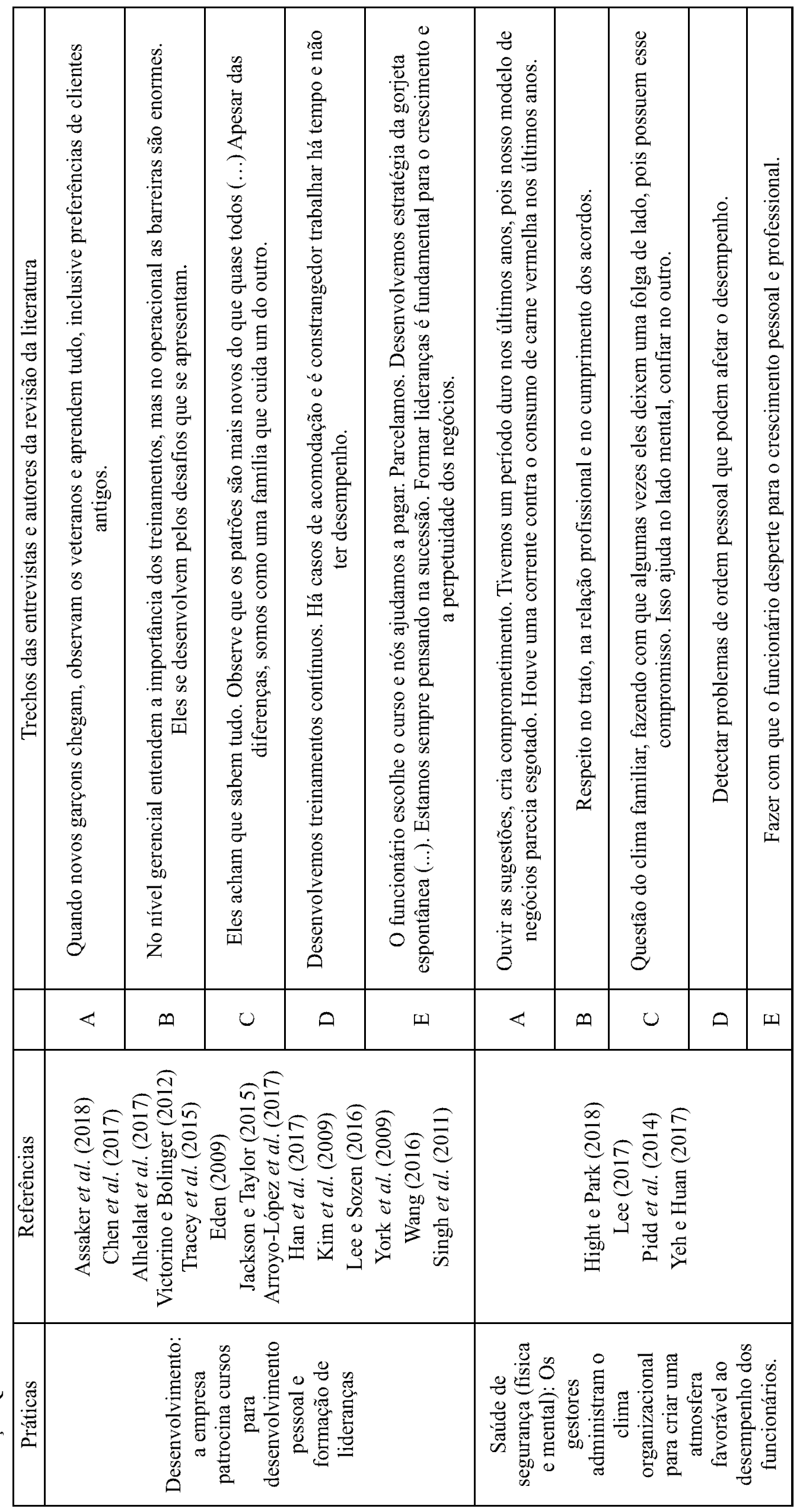


Observa-se o cuidado com o processo de recrutamento, já destacado por Akroush et al. (2013), como a dimensão mais relevante no que diz respeito à equipe. $\mathrm{O}$ recrutamento interno e, mais especificamente, as promoções internas, já eram práticas consolidadas na gestão de restaurantes (MURPHY; OLSEN, 2009). Avançando para discussões além do RH tradicional, a capacidade de contornar conflitos para não demitir sem necessidade, reflete rituais que potencializam o desenvolvimento e a aprendizagem individual e coletiva abordados por Erhardt et al. (2016) dentro do ambiente de trabalho.

O segundo ponto para analisar os dados de aquisição de recursos humanos é a forma de recompensar o trabalho das pessoas. Trata-se de uma estratégia que ajuda a reter bons funcionários e manter elevados seus níveis de produtividade. Pode-se fazer isso por meio de compensações financeiras e não financeiras. Ainda no quadro 2 são apresentados os relatos principais dos gestores dos restaurantes pesquisados com relação às práticas de compensação pelo trabalho executado.

Embora durante as entrevistas tenha ficado clara a diferença na forma de administrar a remuneração, foi possível perceber que os empresários conhecem os fatores que motivam seus profissionais. Na maioria dos estabelecimentos ouvir as sugestões dos funcionários se torna uma prática eficaz. Outra vertente aponta que quando os funcionários demonstram interesse genuíno em progredir, assumindo novas funções e desafios na empresa, eles auferem recompensas proporcionais.

O terceiro ponto relevante do subsistema aquisição é investimento em treinamento e desenvolvimento dos recursos humanos como estratégia para obter vantagens competitivas. No ramo de restaurantes o nível de serviço sempre merece atenção dos gestores, logo os funcionários necessitam de preparo técnico, além de habilidades e competências para lidar com situações adversas.

A preocupação dos gestores com o aprimoramento contínuo dos funcionários é um dos fatores que contribui para a longevidade do negócio. As oportunidades de capacitação oferecidas aos funcionários podem se configurar como fator motivador, reduzindo turnover e contribuindo para a formação de lideranças. Entretanto, os funcionários que não participam dos treinamentos, não aproveitam a oportunidade, não praticam o que aprenderam, ou ainda não alcançam o desempenho desejado mesmo após as capacitações, podem prejudicar o desempenho do negócio. O quadro 2 reflete o cenário relativo às práticas de treinamento e desenvolvimento nos restaurantes objetos deste estudo.

A iniciativa de patrocinar cursos para desenvolvimento pessoal e formação de lideranças reflete uma atividade que potencializa o desenvolvimento e a aprendizagem individual e coletiva, especialmente aqueles que tentam explicar os caminhos para as organizações serem bem-sucedidas, destacadas nos estudos de Erhardt et al. (2016) dentro do ambiente de trabalho.

O quarto e último subsistema de gestão de recursos humanos está ligado à saúde e à segurança física e psíquica do trabalhador. No ramo de restaurantes, as pessoas podem trabalhar de pé, em condições de exposição ao calor e a situações de pressão psicológica. A preocupação com o bemestar dos funcionários deve ser um objetivo a ser alcançado pela empresa, se ela pretende chegar a desempenhos superiores.

Das percepções apresentadas com relação às práticas de gestão da saúde e da segurança dos colaboradores, foi possível sistematizar a prática de administrar o clima organizacional para criar uma atmosfera favorável à saúde mental dos funcionários, evidenciando uma abordagem menos ortodoxa, mas igualmente relevante, que tenta explicar os caminhos para as organizações serem bem-sucedidas. Dentre esses estudos, destacam-se os que se aprofundam em rituais que potencializam o desenvolvimento e a aprendizagem individual e coletiva (ERHARDT et al., 2016) no ambiente de trabalho. 


\subsection{SERVIÇOS TECNOLÓGICOS}

Consumidores exigentes clamam por novidades e, nesse sentido, são válidas as iniciativas que deixam os clientes mais bem informados, por meio de tecnologias que estão nas palmas de suas mãos. Com seus smartphones, eles acessam conteúdos exclusivos e informações gerais que tornam suas experiências de consumo mais completas. Elementos físicos ligados à tecnologia influenciam a satisfação dos clientes. Há empreendimentos que, por exemplo, substituem cardápios físicos por tablets que ficam à disposição do consumidor em algum lugar do restaurante. Esses artefatos tecnológicos dão ar sofisticado ao estabelecimento, melhoram a qualidade da apresentação das informações, acentuam o nível geral de serviços e incrementam as vendas (BELDONA et al., 2014).

Dentre os temas mais pesquisados com relação à gestão de restaurantes com foco na obtenção de vantagens competitivas, estão os estudos que analisam de que forma a comunicação nas redes sociais é útil para propagar a marca de um restaurante e conectar-se aos consumidores de forma barata (DiPIETRO et al., 2012). Há, também, estudos que analisam parcerias entre empreendimentos, por meio de canais online, no intuito de oferecer descontos e vantagens aos consumidores (ZHENG; GUO, 2016).

Atenção especial tem que ser dedicada às self-service technologies (SSTs), cuja base conceitual está focada em entender três aspectos: os atributos intrínsecos e extrínsecos da experiência de SSTs; a influência dessa experiência no comprometimento do consumidor; e o potencial mediador da experiência de consumo transcendental (TCE) com relação a esse mesmo comprometimento com a empresa (WEI et al., 2016).

Com relação aos resultados, apenas o restaurante $C$ relatou não gastar energia para gerenciar recursos tecnológicos que visam a incrementar a experiência do consumidor. Os demais se esforçam para monitorar o relacionamento com o cliente pelas redes sociais.

Essa preocupação se justifica principalmente no que tange às avaliações online pós-consumo. Os donos de restaurantes sabem que essa diligência pode ajudar a recuperar possíveis falhas de serviço e influenciar positivamente a intenção de (re)compra.

O restaurante $\mathrm{D}$ desenvolve práticas mais sofisticadas, pois chega a contratar o sistema de uma empresa para coletar dados e analisar a opinião dos clientes. Assim que concluem a refeição, os consumidores são convidados a responder a pesquisa. Os garçons são treinados para conseguir uma elevada taxa de resposta e, desta forma, se engajam nessa prática, pois sendo bem avaliados, são recompensados pelos gestores. 


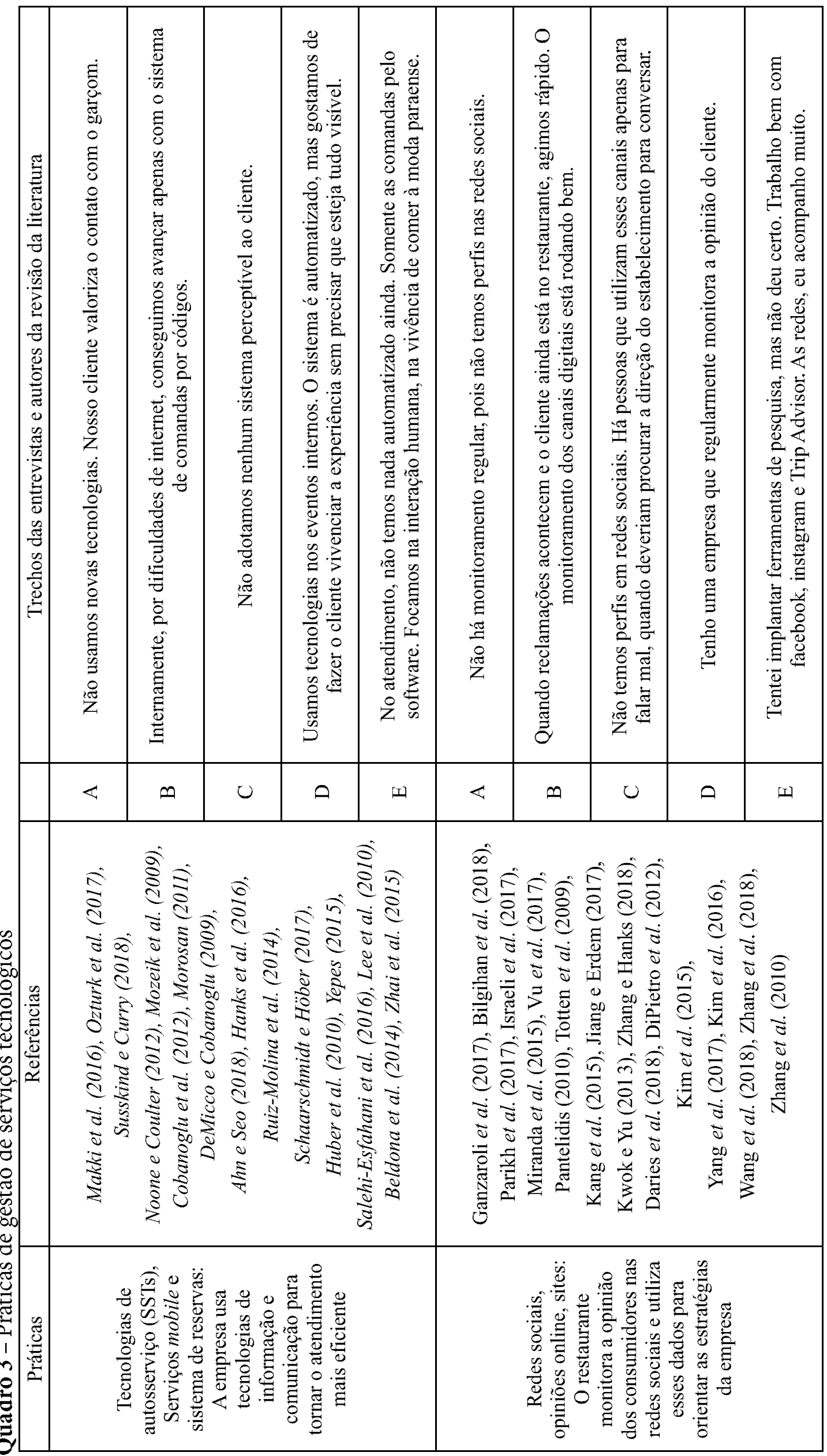


Já o restaurante E incentiva que seus funcionários não se contentem com os protocolares (e polêmicos) $10 \%$ de taxa de serviço, que, no fim das contas, é rateado entre todos os colaboradores. Ele busca promover o encantamento com o serviço prestado na experiência de consumo, de forma que seus garçons ganhem a chamada gorjeta espontânea, ou seja, valores excedentes pagos diretamente aos garçons, de forma voluntária pelo cliente, independentemente do valor que ele consumiu no estabelecimento.

Segundo o gestor do restaurante $\mathrm{E}$, mesmo que o funcionário não receba um valor a título de gorjeta extra, ela prepara seus colaboradores para conseguirem uma prova material de que foram bem atendidos e que recomendam o serviço daquele profissional que os atendeu. Os garçons, então, pedem ao final do atendimento, que se o cliente gostou da experiência de atendimento com ele, que ele tire uma foto com o garçom e poste no Trip Advisor essa evidência e que ele também escreva algo positivo sobre o encontro de serviço.

Dessa forma, diversas referências podem ser registradas nessa plataforma de avaliações on-line, o que configura medidas de bom desempenho que poderão influenciar positivamente potenciais clientes do restaurante, assim como reforçar a intenção de recompra daqueles que já frequentam o estabelecimento.

Já os gestores dos restaurantes B, D e E contam com uma equipe que os ajuda a monitorar o comportamento do consumidor nas redes sociais. Eles chegam ao ponto de investigar se um cliente que manifestou uma insatisfação no ambiente virtual, ainda pode estar no restaurante. Neste caso, eles tentam localizar o cliente e se propõem a recuperar o serviço mais rapidamente, se deixar que o cliente leve para casa a sensação de que não foi atendido a contento.

Todos os gestores reconhecem que as novas tecnologias vieram para ficar e que, cedo ou tarde, os serviços serão todos influenciados por elas. Em alguns casos, elas são a única forma do gestor saber onde precisa melhorar na gestão do restaurante. No entanto, na opinião dos gestores, nem todos os consumidores estão preparados para se comportar adequadamente no ambiente virtual. Por isso, o mau uso das ferramentas tecnológicas desvia o foco daquilo que realmente importa na prestação de serviço dos restaurantes.

\subsection{INTERAÇÃO HUMANA NO ENCONTRO DE SERVIÇOS}

Consumidores de restaurantes têm gostos variados e cada empreendimento busca compreender e satisfazer as necessidades e desejos de seu público. As rotinas de gestão e atendimento se estruturam de forma a atender às expectativas dos clientes de forma eficaz desde o controle do tempo de servir os pratos (KREMER; DEBO, 2016), até como os garçons sugerem pratos e opções de degustação (BARNES et al. 2016).

A arte de equilibrar o novo e o tradicional é difícil de ser dominada. Rotinas necessárias aos restaurantes, tais como padronização de molhos e temperos podem não resultar em capacidades que conferem vantagens competitivas, mas agradam em cheio ao público que valoriza a manutenção de serviços que os agradam ao longo do tempo. Por outro lado, existe um perfil de clientes que clama por novidades e sente que seu dinheiro é mais valorizado quando pode experimentar pratos e formas de servir inéditos (JIN et al., 2016), o que provoca efeitos positivos no seu comprometimento e na sua confiança (NIKBIN et al., 2013).

Essa capacidade de surpreender e cativar os clientes é investigada até no nível que se denomina de alta cozinha ou alta gastronomia. É preciso tanto esforço para chegar ao atendimento das necessidades dos clientes atualmente, que se faz necessário estar em frequente estado de alerta em busca de modelos que levem a novas aprendizagens (ABECASSIS-MOEDAS et al., 2016). 


\begin{tabular}{|c|c|c|c|c|c|c|c|c|c|c|}
\hline 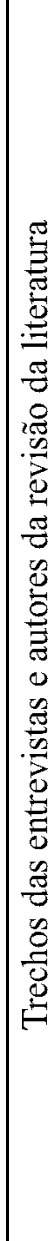 & 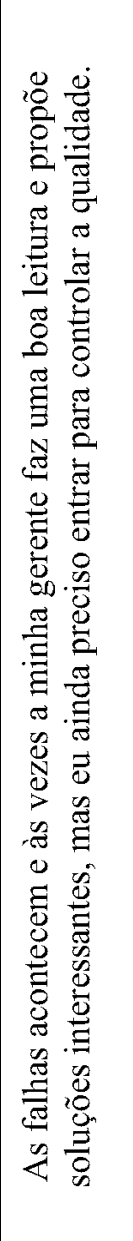 & 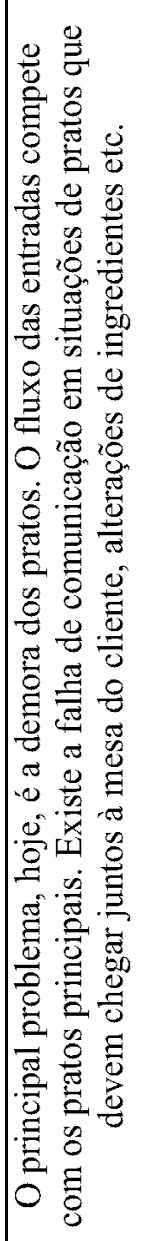 & 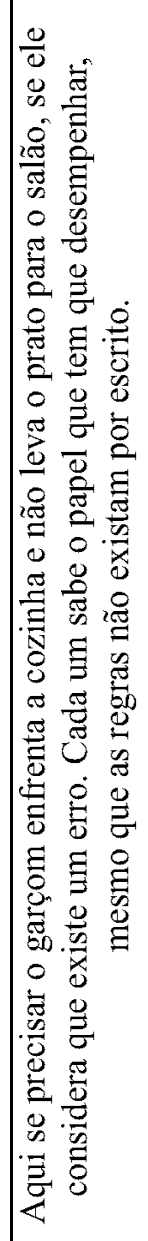 & 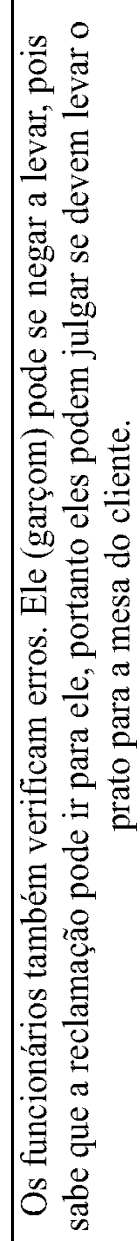 & 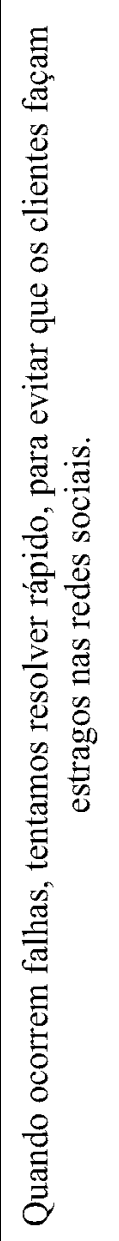 & 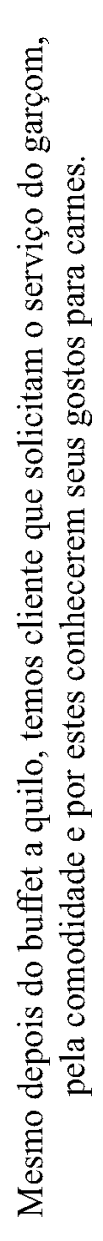 & 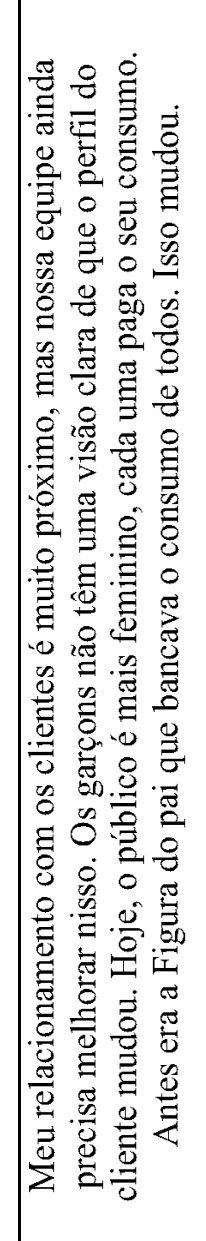 & 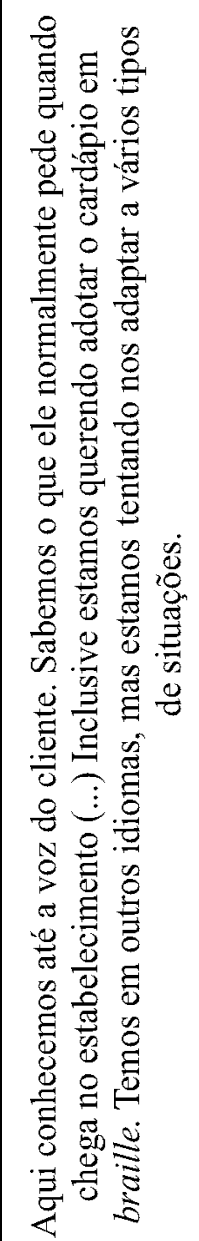 & 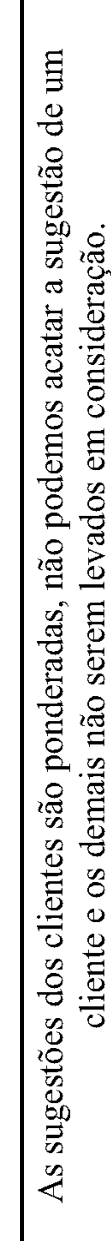 & 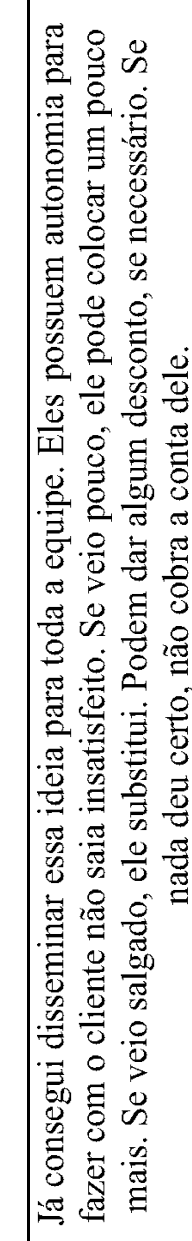 \\
\hline & $\ll$ & $\emptyset$ & $u$ & 0 & 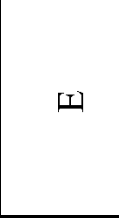 & $<$ & 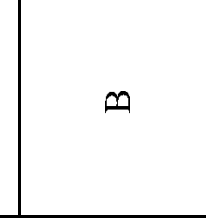 & 0 & 0 & \\
\hline 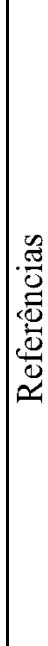 & \multicolumn{5}{|c|}{ 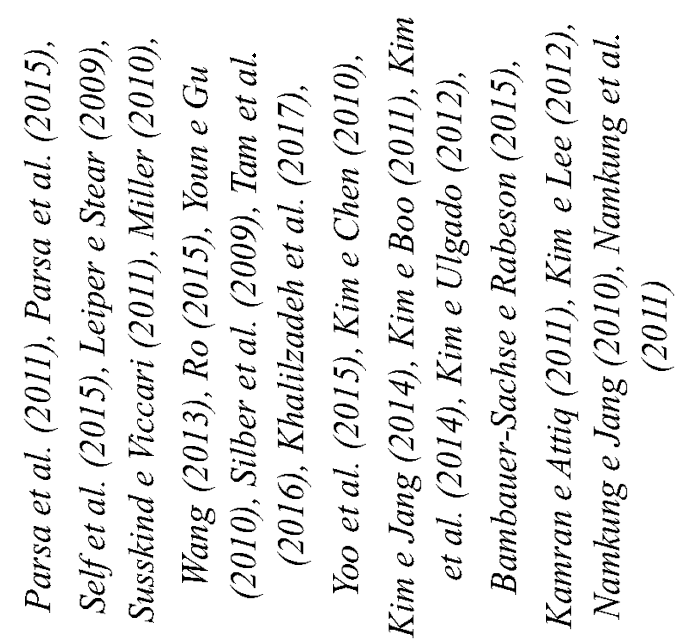 } & \multicolumn{5}{|c|}{ 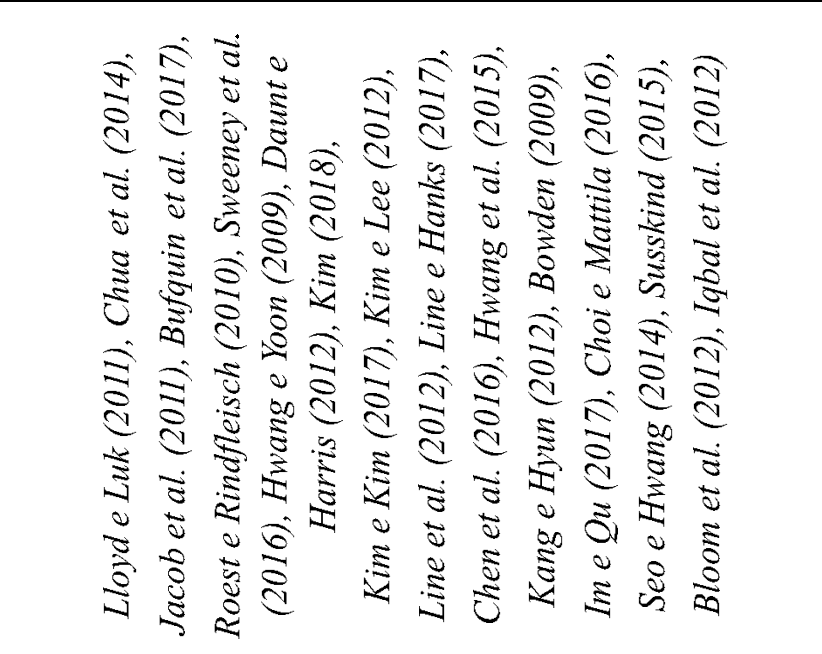 } \\
\hline 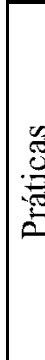 & \multicolumn{5}{|c|}{ 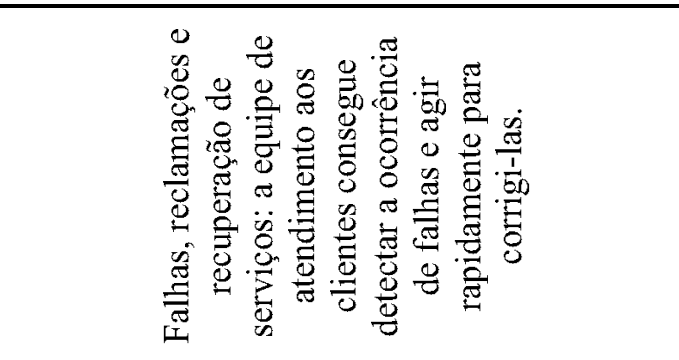 } & \multicolumn{5}{|c|}{ 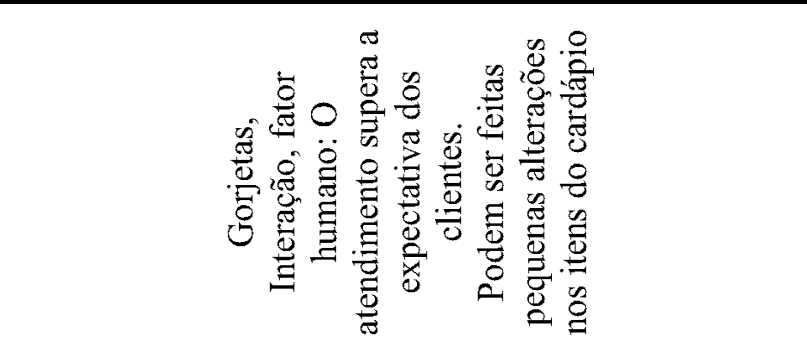 } \\
\hline
\end{tabular}


Outra vertente importante apontada pelos gestores, quase por unanimidade como um elemento diferenciador entre restaurantes bem-sucedidos, é a capacidade do seu pessoal para prestar um bom atendimento. Todos apontaram que o elemento humano ainda é o ponto central da experiência de consumo dentro dos restaurantes.

No restaurante $\mathrm{C}$ essa crença é mais forte que nos demais, pois esse estabelecimento aposta na forma tradicional de servir, na capacidade de oferecer aos clientes um atendimento personalizado, compreendendo os gostos e preferências de consumidores assíduos na casa e surpreendendo positivamente clientes novos e antigos com sugestões de consumo.

De acordo com a revisão da literatura, a presença e o comportamento dos demais clientes e as próprias características do espaço físico onde se dá o encontro de serviços, podem influenciar a experiência de consumo. No entanto, nos casos desses restaurantes paraenses, os gestores não relataram nenhuma prática relacionada a esses aspectos da atmosfera de consumo.

Com relação às falhas e à recuperação de serviços, todos os gestores demonstraram preocupação em contornar problemas que aconteciam no ambiente físico e na maioria dos casos ia resultar em comentários negativos nas redes sociais e sites de registro de experiências de viagens, tal como o Trip Advisor. Os gestores dos restaurantes A e C, apesar de não fazerem grandes investimentos em gestão de redes sociais, se declararam preocupados com os problemas que acontecem por mau atendimento em pedidos por telefone ou então em casos de lotação máxima de seus estabelecimentos.

Eles disseram, inclusive, que quando conseguem detectar o problema com o cliente ainda dentro da empresa, que eles tomam a linha de frente para recuperar o serviço rapidamente. Porém, quando eles tomam conhecimento da falha horas ou dias depois, eles tentam entrar diretamente em contato com os clientes para pedir uma chance de recuperar o serviço.

\subsection{GESTÃO DA SUSTENTABILIDADE}

Nesta dimensão se encontram artigos que tratam de diferentes temas ligados às preocupações com o equilíbrio ambiental, social e econômico dos stakeholders. Isso inclui olhar para elos diferentes da cadeia que abastece e consome produtos das empresas de alimentação fora do lar.

Os clientes de restaurantes podem até fingir ignorância (LEE et al., 2016) para consumir alimentos menos saudáveis sem manifestar sentimento de culpa, mas a verdade é que eles estão cada vez mais atentos e informados (FAKIH et al., 2016). Turistas, por exemplo, ao viajar, buscam informações sobre o destino e também sobre o que terão que encarar nos cardápios dos estabelecimentos onde ele se alimentará durante a estadia (BJÖRK; KAUPPINEN-RÄISÄNEN, 2014; HYUN; PARK, 2015).

Não somente em situações de viagem eles manifestam essa preocupação. As restrições alimentares, as alergias e os cuidados com o corpo têm deixado os consumidores mais exigentes com relação às opções oferecidas na alimentação fora do lar (LOUREIRO; RAHMANI, 2016; LEE; SOZEN, 2016). Os gestores, por outro lado, têm buscado ofertar opções suficientes para essas novas necessidades (LEE et al., 2016).

Com os clientes cada vez mais atentos às origens daquilo que consomem, é necessário se engajar nesse movimento para se manter competitivo. Os restaurantes, então, passaram a se preocupar mais com seus fornecedores, verificando se as práticas de gestão adotadas por eles no cultivo ou na fabricação dos ingredientes são compatíveis com as estratégias do próprio restaurante com relação aos processos de gestão e de comunicação com o mercado. 


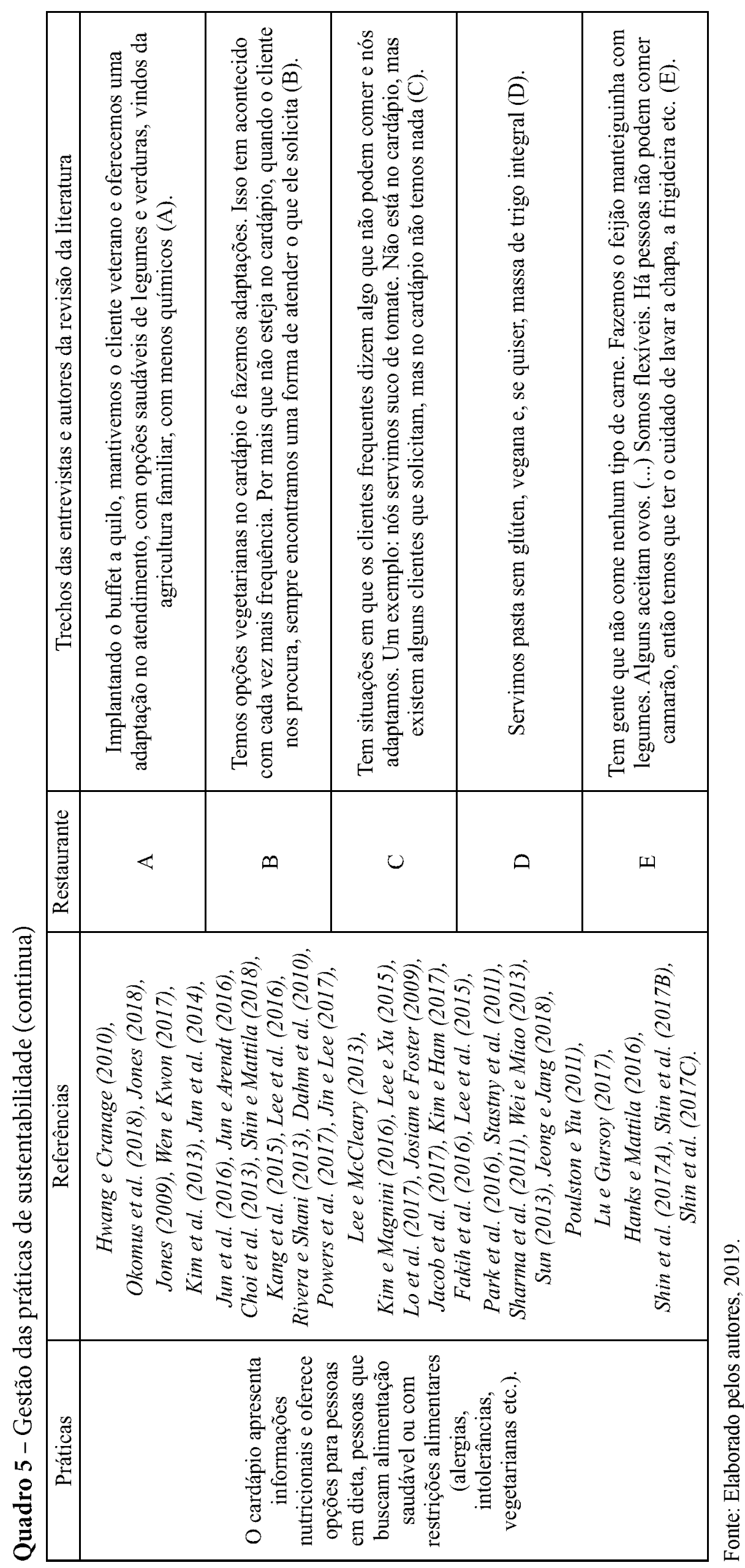




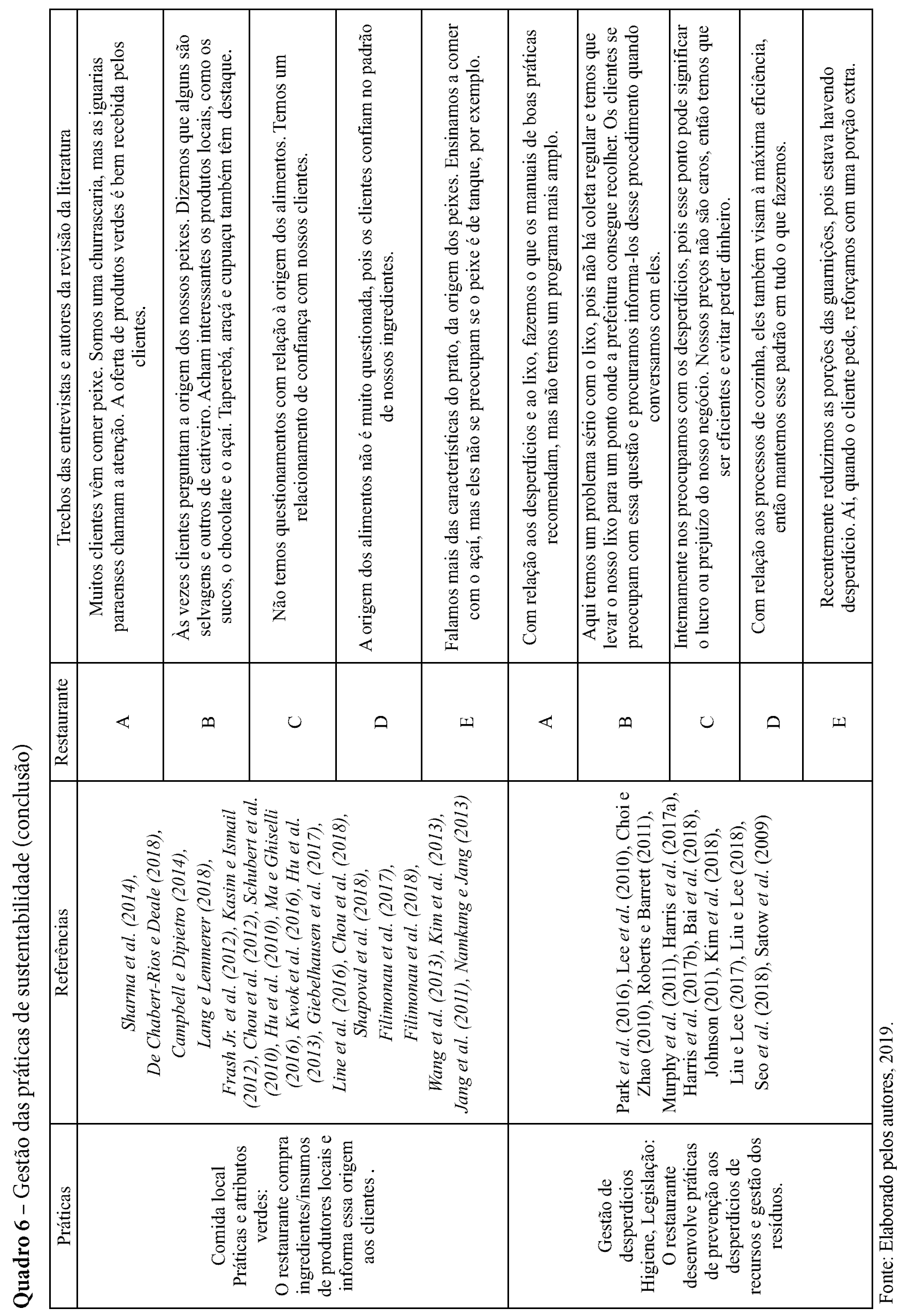


Essa abordagem inclui a oferta de produtos verdes e de produtos que transmitam a ideia de bem estar e saúde; práticas responsáveis e justas com relação ao tipo de negociação feita com fornecedores; atitudes inteligentes com relação ao aproveitamento dos recursos naturais e materiais empregados na produção de alimentos, evitando desperdícios; a busca a melhor procedência de insumos; enfim, práticas que, hoje, são valorizadas pelos consumidores e, por vezes, utilizadas como estratégia para atrair clientes (RHOU et al., 2016; LINE et al., 2016).

Os resultados relacionados às práticas de gestão da sustentabilidade envolvem rotinas de relacionamento com stakeholders, com relação ao equilíbrio social, ambiental e econômico nesses laços. Com relação à origem dos insumos, os restaurantes A e B relataram seus clientes costumam ter essa preocupação. Os restaurantes $\mathrm{C}$ e D afirmaram que seus clientes não manifestam esse interesse por terem uma relação de confiança com o estabelecimento e acreditarem que eles fazem as escolhas certas. Já os clientes do restaurante E não se prendem a esse detalhe, pois, na maioria dos casos, estão mais interessados em aproveitar a experiência de combinar os ingredientes regionais, prestando mais atenção aos sabores em si.

Outro grupo importante de práticas está ilustrado no Quadro 5 e está ligado a algumas ações que o estabelecimento tem que fazer para se adaptar às preferências do consumidor. Para o restaurante A, essa adaptação foi profunda e chegou a alterar a forma de atendimento aos clientes. Nos demais estabelecimentos, as adaptações acontecem mais com relação troca de ingredientes, tamanho de porções e ofertas de alguns itens que não são de conhecimento do público em geral, apenas dos clientes mais fiéis.

Um dos principais pontos de atuação tem sido as mudanças estruturais de cardápio, introduzindo opções de pratos e ingredientes para clientes que apresentam restrições alimentares ligadas à saúde como alergias e intolerâncias - e também o público que busca fora do lar, por necessidade ou adoção de um padrão de vida mais saudável, consumir alimentos mais nutritivos e que ajudam a melhorar o funcionamento de seu organismo.

No que tange aos processos de produção, prevenção de desperdícios e tratamento dos resíduos proveniente dessa atividade, os restaurantes foram quase unânimes em afirmar que não possuem programas específicos para reduzir os impactos que isso pode causar no financeiro da empresa e no nomeio ambiente. Eles relataram seguir os manuais de boas práticas, exigido por lei, mas não citaram isso como uma preocupação de seus clientes. A exceção ficou por conta do restaurante B, que se localiza em uma área onde não há coleta de lixo regular, o que exige de sua equipe dedicação para evitar ao máximo o aumento de desperdícios. Da mesma forma, o descarte dos resíduos de sua produção são foco de atenção tanto do empreendimento quanto dos clientes que frequentam o espaço.

\section{CONCLUSÕES}

Após a identificação das práticas de gestão mais estudadas na literatura recente na área de hospitalidade, por meio da análise dos 381 artigos, verificou-se a aderência dessas práticas ao contexto paraense a partir da análise dos conteúdos das entrevistas com os gestores de cinco restaurantes da cidade de Belém do Pará.

Os resultados apontam para a abertura de agenda de pesquisa sobre práticas de gestão em restaurantes, com foco na investigação das rotinas desses estabelecimentos e na consolidação de práticas já consagradas e que podem explicar diferenças de desempenho entre os restaurantes, assim como chegar a novos conjuntos de práticas para esta mesma finalidade. 
As implicações gerenciais mais relevantes da presente pesquisa estão ligadas à compreensão de quais práticas estão alinhadas às rotinas de gestão desses estabelecimentos em nível mundial, para que suas estruturas física, tecnológica e de RH possam ser preparadas para a prestação do serviço de alimentação fora do lar.

Na dimensão de práticas de gestão estratégica de RH, no subsistema de aquisição, as práticas apontaram rotinas de manutenção de baixa rotatividade e preferência por recrutamento interno. No subsistema compensação, a busca por construção de elos de confiança a partir da forma com que os funcionários são tratados e pela gestão participativa, à medida que o tempo de casa os alça à condição de líderes que sugerem e auxiliam diretamente na gestão. No subsistema desenvolvimento há dificuldades, pois a elevada experiência média dos funcionários gera comodismo e resistência a novos aprendizados. Apenas duas das empresas conseguem desenvolver treinamentos contínuos, sendo uma com rotinas internas e outra busca fora da empresa essa capacitação aos funcionários. Por fim, no subsistema saúde e segurança, as práticas estão voltadas aos cuidados com a saúde psicológica quando se verifica que as rotinas se concentram na manutenção de um bom clima organizacional, cuidando do lado mental e emocional das pessoas, gerando compromissos mútuos.

Na dimensão de práticas de gestão de serviços tecnológicos, verificou-se o uso mais intenso de tecnologias no gerenciamento dos pedidos dos clientes no salão e nos sistemas de reservas. Já o monitoramento sistemático dos perfis e das manifestações dos clientes nas redes sociais se apresentou como rotinas fortemente presentes no cotidiano dos restaurantes pesquisados, ressaltando que eles apresentam dificuldades para consolidar seus próprios instrumentos online de medida de satisfação dos clientes.

Na dimensão de práticas de gestão a interação humana no encontro de serviços, destaca-se o foco na detecção e na recuperação de falhas, com destaque para a autonomia que os gestores procuram dar aos garçons para tomar as medidas necessárias que garantam a satisfação dos clientes. A experiência dos atendentes faz até com que falhas sejam evitadas, mas houve restaurantes que relataram resistências ao entendimento de que o perfil do consumidor passou por transformações radicais nos últimos anos.

Por fim, na dimensão de práticas de gestão da sustentabilidade destacam-se as adaptações recentes feitas nos cardápios e nos processos de preparo de alimentos com relação à busca por uma alimentação mais saudável e ao aumento do número de pessoas alérgicas a determinados alimentos, embora nem todos os gestores tenham conseguido incluir no menu essas opções. Eles ainda agem reativamente quando demandados pelos clientes. A preocupação com a origem dos ingredientes dos pratos ainda não se mostrou forte, pois os gestores relataram que os clientes confiam nas escolhas dos estabelecimentos. As perguntas sobre este tema ainda aparecem mais a título de curiosidade do que algo que restrinja o consumo. Por sua vez, as práticas relacionadas à gestão dos resíduos e à prevenção dos desperdícios apresentaram viés ambiental no caso de um dos restaurantes que se localiza em uma área de proteção ambiental, enquanto para os demais os relatos se referem à questão de custos, que pode levá-los a melhores resultados financeiros.

Como a etapa qualitativa foi realizada apenas com cinco estabelecimentos localizados em Belém do Pará, um dos avanços necessários para estudos futuros é a inclusão de um número maior estabelecimentos, locais e de outros estados brasileiros, aumentando as chances de verificar esta aderência de forma mais eficaz e abrangente.

Questões importantes para a área de estratégia foram propositalmente deixadas de lado neste estudo, como as discussões sobre branding, responsabilidade social corporativa, finanças e contabilidade, questões étnicas, entre outras. Estes tópicos aparecem na agenda dos pesquisadores 
de práticas de gestão em restaurantes, mas foram preteridos em razão das escolhas metodológicas e dos recursos para executar a pesquisa. Há, portanto, espaço para aprofundamento dessas questões em âmbito local, regional e nacional, partindo de escolhas de desenho de pesquisa voltados para esses fins.

Apesar das limitações, o presente estudo contribui no sentido de aproximar a realidade de mercado dos restaurantes paraenses às pesquisas acadêmicas de vanguarda. Mesmo em empresas familiares, com dezenas de anos de tradição, práticas de gestão são relacionadas a rotinas analisadas em diferentes países.

\section{REFERÊNCIAS}

ABECASSIS-MOEDAS, C.; SGUERA, F.; ETTLIE, J. E. Observe, innovate, succeed: a learning perspective on innovation and the performance of entrepreneurial chefs. Journal of Business Research, v. 69, n. 8, pp. 2840-2848, 2016.

AHN, Jee Ahe; SEO, Soobin. Consumer responses to interactive restaurant self-service technology (IRSST): The role of gadget-loving propensity. International Journal of Hospitality Management, v. 74, p. 109-121, 2018

AKROUSH, M. N.; ABU-El SAMEN, A. A.; SAMAWI, G. A.; ODETALLAH, A. L. Internal marketing and service quality in restaurants. Marketing Intelligence \& Planning. v. 31, n. 4, p. 304-336, 2013.

ALHELALAT, Jebril A.; MA’MOUN, A. Habiballah; TWAISSI, Naseem M. The impact of personal and functional aspects of restaurant employee service behaviour on customer satisfaction.

International Journal of Hospitality Management, v. 66, p. 46-53, 2017.

ARIFFIN, H. F.; BIBON, M. F.; ABDULLAH, R. P. S. R. Restaurant's atmospheric elements: What the customer wants. Procedia-Social and Behavioral Sciences, v. 38, pp. 380-387, 2012.

ARROYO-LÓPEZ, Pilar Ester et al. Impact of training on improving service quality in small provincial restaurants. Journal of Foodservice Business Research, v. 20, n. 1, p. 1-14, 2017.

ASSAKER, Guy; HALLAK, Rob; O'CONNOR, Peter. Examining heterogeneity through responsebased unit segmentation in PLS-SEM: a study of human capital and firm performance in upscale restaurants. Current Issues in Tourism, p. 1-16, 2018.

BAI, Li et al. Food safety in restaurants: The consumer perspective. International Journal of Hospitality Management, v. 77, p. 139-146, 2018.

BAMBAUER-SACHSE, Silke; RABESON, Landisoa Eunorphie. Service recovery for moderate and high involvement services. Journal of Services Marketing, v. 29, n. 5, p. 331-343, 2015.

BARNES, D. C.; MEYER, T.; KINARD, B. R. Implementing a delight strategy in a restaurant setting: the Power of unsolicited recommendations. Cornell Hospitality Quarterly, v. 57, p. 329-342, 2016.

BELDONA, Srikanth; BUCHANAN, Nadria; MILLER, Brian L. Exploring the promise of e-tablet restaurant menus. International Journal of Contemporary Hospitality Management. v. 26, n. 3, p. 367-382, 2014. 
BILGIHAN, Anil; SEO, Soobin; CHOI, Jihee. Identifying restaurant satisfiers and dissatisfiers: Suggestions from online reviews. Journal of Hospitality Marketing \& Management, v. 27, n. 5, p. 601-625, 2018.

BJÖRK, P.; KAUPPINEN-RÄISÄNEN, H. Exploring the multi-dimensionality of travellers' culinary-gastronomic experiences. Current Issues in Tourism, v. 19, n. 12, p. 1260-1280, 2016.

BLOOM, Barry AN et al. The impact of meal duration on a corporate casual full-service restaurant chain. Journal of foodservice business research, v. 15, n. 1, p. 19-38, 2012.

BOWDEN, Jana. Customer engagement: A framework for assessing customer-brand relationships: The case of the restaurant industry. Journal of Hospitality Marketing \& Management, v. 18, n. 6, p. 574-596, 2009.

BUFQUIN, Diego et al. Effects of social perceptions and organizational commitment on restaurant performance. Journal of Hospitality Marketing \& Management, v. 26, n. 7, p. 752-769, 2017.

BUFQUIN, Diego et al. The influence of restaurant co-workers' perceived warmth and competence on employees' turnover intentions: The mediating role of job attitudes. International Journal of Hospitality Management, v. 60, p. 13-22, 2017.

CAMPBELL, Jeffrey M.; DIPIETRO, Robin B. Sign of the times: Testing consumer response to local food signage within a casual dining restaurant. Journal of Retailing and Consumer Services, v. 21, n. 5, p. 812-823, 2014.

CHEN, Yang-Su et al. Investigating children's role in family dining-out choices: Evidence from a casual dining restaurant. Journal of Hospitality Marketing \& Management, v. 25, n. 6, p. 706-725, 2016.

CHEN, Yang-Su; RAAB, Carola; CHEN, Chih-Chien. The influence of celebrity chefs on restaurant customers' behavior. Journal of Hospitality Marketing \& Management, v. 26, n. 5, p. 489-510, 2017.

CHOI, Choongbeom; MATTILA, Anna S. The effects of other customers' dress style on customers' approach behaviors: the moderating role of sense of power. Cornell Hospitality Quarterly, v. 57, n. 2, p. 211-218, 2016.

CHOI, Jinkyung et al. Consumers' responses to restaurant inspection reports: The effects of information source and message style. Journal of foodservice business research, v. 16, n. 3, p. 255-275, 2013.

CHOI, Jinkyung; ZHAO, Jinlin. Factors influencing restaurant selection in south florida: Is health issue one of the factors influencing consumers' behavior when selecting a restaurant? Journal of Foodservice Business Research, v. 13, n. 3, p. 237-251, 2010.

CHOI, Kyuwan; HWANG, Johye; PARK, Myoungju. Scheduling restaurant workers to minimize labor cost and meet service standards. Cornell Hospitality Quarterly, v. 50, n. 2, p. 155-167, 2009. CHOU, Chia-Jung; CHEN, Kuo-Sheng; WANG, Yueh-Ying. Green practices in the restaurant industry from an innovation adoption perspective: Evidence from Taiwan. International Journal of Hospitality Management, v. 31, n. 3, p. 703-711, 2012. 
CHOU, Sheng-Fang et al. Explicating restaurant performance: The nature and foundations of sustainable service and organizational environment. International Journal of Hospitality Management, v. 72, p. 56-66, 2018.

CHUA, Bee-Lia et al. Influence of mechanic, functional, and humanic clues on customers' experiential values and behavioral intentions in full-service restaurants. Journal of foodservice business research, v. 17, n. 2, p. 67-84, 2014.

COBANOGLU, Cihan et al. The impact of Wi-Fi service in restaurants on customers' likelihood of return to a restaurant. Journal of Foodservice Business Research, v. 15, n. 3, p. 285-299, 2012.

DAHM, Molly J.; SHOWS, Amy R.; SAMONTE, Aurelia V. Eating behaviors, obesity, and litigation: should casual-food restaurant operators heed the warnings to their fast-food counterparts? Journal of Foodservice Business Research, v. 13, n. 3, p. 217-236, 2010.

DARIES, Natalia et al. Maturity and development of high-quality restaurant websites: A comparison of Michelin-starred restaurants in France, Italy and Spain. International Journal of Hospitality Management, v. 73, p. 125-137, 2018.

DAUNT, Kate L.; HARRIS, Lloyd C. Motives of dysfunctional customer behavior: an empirical study. Journal of Services Marketing, v. 26, n. 4, p. 293-308, 2012.

DE CHABERT-RIOS, Jacqueline; DEALE, Cynthia S. Taking the local food movement one step further: an exploratory case study of hyper-local restaurants. Tourism and Hospitality Research, v. 18, n. 3, p. 388-399, 2018.

DEMICCO, Fred J.; COBANOGLU, Cihan. The Microsoft home: applications for the lodging and restaurant industry today and in the future. Journal of Foodservice Business Research, v. 12, n. 1, p. 95-106, 2009.

DIPIETRO, R. B.; CREWS, T. B.; GUSTAFSON, C; STRICK, S. The use of social networking sites in the restaurant industry: best practices. Journal of Food Service Business Research, v. 15, n. 3, p. 265-284, 2012.

DURRANI, Amir S.; RAJAGOPAL, Lakshman. Restaurant human resource managers' attitudes towards workplace diversity, perceptions and definition of ethical hiring. International Journal of Hospitality Management, v. 53, p. 145-151, 2016.

EDEN, Jason. Research Note: How Routinization Affects Power Dynamics at a Family Restaurant Chain. Journal of Foodservice Business Research, v. 12, n. 1, p. 84-94, 2009.

ERHARDT, N; MARTIN-RIOS, C; HECKSCHER, C. Am I doing the right thing? Unpacking workplace rituals as mechanisms for strong organizational culture. International Journal of Hospitality Management, v.59, p. 31-41, 2016.

FAKIH, K.; ASSAKER, G.; ASSAF, A. G.; HALLAK, R. Does restaurant menu information affect customer attitudes and behavioral intentions? A cross-segment empirical analysis using PLS-SEM. International Journal of Hospitality Management, v. 57, p. 71-83, 2016. 
FILIMONAU, Viachaslau et al. Restaurant menu re-design as a facilitator of more responsible consumer choice: An exploratory and preliminary study. Journal of Hospitality and Tourism Management, v. 33, p. 73-81, 2017.

FILIMONAU, Viachaslau et al. The determinants of more responsible restaurant food choice in Poland. Journal of Sustainable Tourism, v. 26, n. 8, p. 1398-1416, 2018

FRASH JR, Robert E. Eat, drink, and tip: Exploring economic opportunities for full-service restaurants. Journal of foodservice business research, v. 15, n. 2, p. 176-194, 2012.

GANZAROLI, Andrea; DE NONI, Ivan; VAN BAALEN, Peter. Vicious advice: Analyzing the impact of TripAdvisor on the quality of restaurants as part of the cultural heritage of Venice. Tourism Management, v. 61, p. 501-510, 2017.

GIEBELHAUSEN, Michael et al. The warm glow of restaurant checkout charity. Cornell Hospitality Quarterly, v. 58, n. 4, p. 329-341, 2017

HAN, Su Jin; BONN, Mark A.; CHO, Meehee. The relationship between customer incivility, restaurant frontline service employee burnout and turnover intention. International Journal of Hospitality Management, v. 52, p. 97-106, 2016.

HAN, Su Jin; KIM, Woo Gon; KANG, Sora. Effect of restaurant manager emotional intelligence and support on front-of-house employees' job satisfaction. International Journal of Contemporary Hospitality Management, v. 29, n. 11, p. 2807-2825, 2017.

HANKS, Lydia; LINE, Nathan D.; MATTILA, Anna S. The impact of self-service technology and the presence of others on cause-related marketing programs in restaurants. Journal of Hospitality Marketing \& Management, v. 25, n. 5, p. 547-562, 2016.

HANKS, Lydia; MATTILA, Anna S. Consumer response to organic food in restaurants: A serial mediation analysis. Journal of Foodservice Business Research, v. 19, n. 1, p. 109-121, 2016.

HARRIS, Kimberly J. et al. The antecedents and outcomes of food safety motivators for restaurant workers: An expectancy framework. International Journal of Hospitality Management, v. 63, p. 53-62, 2017b.

HARRIS, Kimberly J. et al. Understanding responses to posted restaurant food safety scores: An information processing and regulatory focus perspective. International Journal of Hospitality Management, v. 60, p. 67-76, 2017a.

HIGHT, S. Kyle; PARK, Jeong-Yeol. Substance use for restaurant servers: Causes and effects. International Journal of Hospitality Management, v. 68, p. 68-79, 2018.

HU, Hsin-Hui; PARSA, H. G.; SELF, John. The dynamics of green restaurant patronage. Cornell Hospitality Quarterly, v. 51, n. 3, p. 344-362, 2010.

HUBER, Marsha M.; HANCER, Murat; GEORGE, R. Thomas. A comparative examination of information technology usage in the restaurant industry. Journal of Foodservice Business Research, v. 13, n. 3, p. 268-281, 2010. 
HWANG, Jinsoo; HAN, Heesup; KIM, Seongseop. How can employees engage customers? Application of social penetration theory to the full-service restaurant industry by gender.

International Journal of Contemporary Hospitality Management, v. 27, n. 6, p. 1117-1134, 2015.

HWANG, Johye; YOON, So-Yeon. Where would you like to sit? Understanding customers' privacy-seeking tendencies and seating behaviors to create effective restaurant environments. Journal of Foodservice Business Research, v. 12, n. 3, p. 219-233, 2009.

HWANG, Jungjin; CRANAGE, David. Customer health perceptions of selected fast-food restaurants according to their nutritional knowledge and health consciousness. Journal of Foodservice Business Research, v. 13, n. 2, p. 68-84, 2010.

HYUN, S. S.; PARK, S. H. The antecedents and consequences of travelers' need for uniqueness: an empirical study of restaurant experiences. Asia Pacific Journal of Tourism Research, v. 21, n. 6, pp. 596-623, 2016.

IM, Jinyoung; QU, Hailin. Drivers and resources of customer co-creation: A scenario-based case in the restaurant industry. International Journal of Hospitality Management, v. 64, p. 31-40, 2017.

IQBAL, Qamar; WHITMAN, Lawrence E.; MALZAHN, Don. Reducing customer wait time at a fast food restaurant on campus. Journal of foodservice business research, v. 15, n. 4, p. 319-334, 2012.

ISRAELI, A. Aviad; LEE, Seonjeong Ally; KARPINSKI, Aryn C. Investigating the dynamics and the content of customers' social media reporting after a restaurant service failure. Journal of Hospitality Marketing and Management, v. 26, n. 6, p. 606-626, 2017.

IVANCEVICH, J.M. Gestão de recursos humanos. São Paulo: McGraw-Hill, 2008.

JACKSON, Leonard A.; TAYLOR, Marcia. Revisiting smoking bans in restaurants: Canadian employees perspectives. Tourism and Hospitality Research, v. 15, n. 2, p. 91-104, 2015.

JACOB, Céline; BOULBRY, Gaëlle; GUÉGUEN, Nicolas. Does the information regarding the ingredients composing a dish influence consumers' decisions? An evaluation in a restaurant. Journal of Hospitality Marketing \& Management, v. 26, n. 2, p. 207-214, 2017.

JACOB, Céline; GUÉGUEN, Nicolas; BOULBRY, Gaelle. Presence of various figurative cues on a restaurant table and consumer choice: Evidence for an associative link. Journal of Foodservice Business Research, v. 14, n. 1, p. 47-52, 2011.

JACQUES, Paul H. et al. Authentic leadership on the frontline and its effects on Korean restaurant employees. Journal of Foodservice Business Research, v. 18, n. 4, p. 389-403, 2015.

JANG, Yoon Jung; KIM, Woo Gon; BONN, Mark A. Generation Y consumers' selection attributes and behavioral intentions concerning green restaurants. International Journal of Hospitality Management, v. 30, n. 4, p. 803-811, 2011.

JEONG, EunHa; JANG, SooCheong Shawn. Price premiums for organic menus at restaurants: What is an acceptable level? International Journal of Hospitality Management, v. 77, p. 117127, 2018. 
JIANG, Lan; ERDEM, Mehmet. Twitter-marketing in multi-unit restaurants: Is it a viable marketing tool? Journal of foodservice business research, v. 20, n. 5, p. 568-578, 2017.

JIN, N.; LINE, N. D.; MERKEBU, J. Examining the impact of consumer innovativeness and innovative restaurant image in upscale restaurants. Cornell Hospitality Quarterly, v. 57, n. 3, pp. 268-281, 2016.

JIN, Naehyun; LEE, Sang-Mook. A conceptual framework for healthy food choice in full-service restaurant. Journal of Foodservice Business Research, v. 20, n. 3, p. 304-320, 2017.

JOHNSON, Mark. An End User Perspective: The Impact of FSMA on Restaurants. Cornell Hospitality Quarterly, v. 59, n. 1, p. 85-92, 2018.

JONES, Cathleen S. Taking up space? How customers react to health information and health icons on restaurant menus. Journal of Foodservice Business Research, v. 12, n. 4, p. 344-363, 2009.

JONES, Cathleen. Restaurant food choices by moms: An exploratory study. Journal of foodservice business research, v. 21, n. 4, p. 377-393, 2018.

JOSIAM, Bharath; FOSTER, Charles. Nutritional information on restaurant menus: Who cares and why restauranteurs should bother. International Journal of Contemporary Hospitality Management, v. 21, n. 7, p. 876-891, 2009.

JUN, Jinhyun; ARENDT, Susan W. Understanding healthy eating behaviors at casual dining restaurants using the extended theory of planned behavior. International Journal of Hospitality Management, v. 53, p. 106-115, 2016.

JUN, Jinhyun; ARENDT, Susan W.; KANG, Juhee. Understanding customers' healthful food selection at restaurants: Roles of attitude, gender, and past experience. Journal of Foodservice Business Research, v. 19, n. 2, p. 197-212, 2016.

JUN, Jinhyun; KANG, Juhee; ARENDT, Susan W. The effects of health value on healthful food selection intention at restaurants: considering the role of attitudes toward taste and healthfulness of healthful foods. International Journal of Hospitality Management, v. 42, p. 85-91, 2014.

KAMRAN, Sohail; ATTIQ, Madiha. Value recovery with customer dissatisfaction: A study of restaurant services in Pakistan. Tourism and Hospitality Research, v. 11, n. 3, p. 169-180, 2011.

KANG, Juhee; HYUN, Sunghyup Sean. Effective communication styles for the customer-oriented service employee: Inducing dedicational behaviors in luxury restaurant patrons. International Journal of Hospitality Management, v. 31, n. 3, p. 772-785, 2012.

KANG, Juhee; JUN, Jinhyun; ARENDT, Susan W. Understanding customers' healthy food choices at casual dining restaurants: Using the Value-Attitude-Behavior model. International Journal of Hospitality Management, v. 48, p. 12-21, 2015.

KANG, Juhee; TANG, Liang; FIORE, Ann Marie. Restaurant brand pages on Facebook: Do active member participation and monetary sales promotions matter? International Journal of Contemporary Hospitality Management, v. 27, n. 7, p. 1662-1684, 2015. 
KASIM, Azilah; ISMAIL, Anida. Environmentally friendly practices among restaurants: drivers and barriers to change. Journal of Sustainable Tourism, v. 20, n. 4, p. 551-570, 2012.

KEITH, N. K.; SIMMERS, C. S. Measuring Service Quality Perceptions of Restaurant Experiences: The Disparity Between Comment Cards and DINESERV, Journal of Foodservice Business Research, v. 14, n.1, p. 20-32, 2011.

KHALILZADEH, Jalayer; GHAHRAMANI, Ladan; TABARI, Saloomeh. From "Hypercritics" to "Happy Campers": Who Complains the Most in Fine Dining Restaurants? Journal of Hospitality Marketing \& Management, v. 26, n. 5, p. 451-473, 2017.

KIM, Eojina; HAM, Sunny. Development and validation of a measure of consumer behaviors toward nutritional labeling in restaurants. Journal of foodservice business research, v. 20, n. 5, p. 595-610, 2017.

KIM, Hyun Jeong et al. Does perceived restaurant food healthiness matter? Its influence on value, satisfaction and revisit intentions in restaurant operations in South Korea. International Journal of Hospitality Management, v. 33, p. 397-405, 2013.

KIM, Jong-Hyeong; CHEN, Joseph S. The effects of situational and personal characteristics on consumer complaint behavior in restaurant services. Journal of Travel \& Tourism Marketing, v. 27, n. 1, p. 96-112, 2010.

KIM, Jong-Hyeong; JANG, SooCheong Shawn. The fading affect bias: Examining changes in affect and behavioral intentions in restaurant service failures and recoveries. International Journal of Hospitality Management, v. 40, p. 109-119, 2014.

KIM, Jooho; BOO, Soyoung. Influencing factors on customers' intention to complain in a franchise restaurant. Journal of Hospitality Marketing \& Management, v. 20, n. 2, p. 217-237, 2011.

KIM, Kwon-Soo. The effects of interpersonal attraction on service justice. Journal of Services Marketing, v. 32, n. 6, p. 728-738, 2018.

KIM, Lisa Hyunjung; KIM, Dong Jin. Achieving relational outcomes in casual dining restaurants through consumer commitment. Current issues in tourism, v. 20, n. 2, p. 178-203, 2017.

KIM, Min Gyung; LEE, Chung Hun; MATTILA, Anna S. Determinants of customer complaint behavior in a restaurant context: The role of culture, price level, and customer loyalty. Journal of Hospitality Marketing \& Management, v. 23, n. 8, p. 885-906, 2014.

KIM, Min Gyung; YANG, Hyunjoo; MATTILA, Anna S. The Impact of Customer Loyalty and Restaurant Sanitation Grades on Revisit Intention and the Importance of Narrative Information: The Case of New York Restaurant Sanitation Grading System. Cornell Hospitality Quarterly, v. 59, n. 3, p. 275-284, 2018.

KIM, Namin; LEE, Moonkyu. Other customers in a service encounter: examining the effect in a restaurant setting. Journal of Services Marketing, v. 26, n. 1, p. 27-40, 2012.

KIM, Namin; ULGADO, Francis M. The effect of on-the-spot versus delayed compensation: the moderating role of failure severity. Journal of Services Marketing, v. 26, n. 3, p. 158-167, 2012. 
KIM, Seongseop; LEE, Jin-Soo. Is satisfaction enough to ensure reciprocity with upscale restaurants? The role of gratitude relative to satisfaction. International Journal of Hospitality Management, v. 33, p. 118-128, 2013.

KIM, Seontaik; MAGNINI, Vincent P. Prompting restaurant diners to eat healthy: Atmospheric and menu-related factors. Journal of Foodservice Business Research, v. 19, n. 3, p. 236-254, 2016.

KIM, Seunghyun et al. Effects of social media on firm value for US restaurant companies.

International Journal of Hospitality Management, v. 49, p. 40-46, 2015.

KIM, Taegoo; JUNG-EUN YOO, Joanne; LEE, Gyehee. Post-recovery customer relationships and customer partnerships in a restaurant setting. International Journal of Contemporary Hospitality Management, v. 24, n. 3, p. 381-401, 2012.

KIM, Wansoo; OK, Chihyung; LEE, Myong Jae. Antecedents of service employees' organizational citizenship behaviors in full-service restaurants in Korea. Cornell Hospitality Quarterly, v. 50, n. 2, p. 180-197, 2009.

KIM, Woo Gon; LI, Jun Justin; BRYMER, Robert A. The impact of social media reviews on restaurant performance: The moderating role of excellence certificate. International Journal of Hospitality Management, v. 55, p. 41-51, 2016.

KIM, Yong Joong; NJITE, David; HANCER, Murat. Anticipated emotion in consumers' intentions to select eco-friendly restaurants: Augmenting the theory of planned behavior. International Journal of Hospitality Management, v. 34, p. 255-262, 2013.

KREMER, M.; DEBO, L. Inferring quality from wait time. Management Science, v. 62, n. 10, p. 3023-3038, 2016.

KWOK, Linchi; HUANG, Yung-Kuei; HU, Lanlan. Green attributes of restaurants: what really matters to consumers? International Journal of Hospitality Management, v. 55, pp. 107-117, 2016.

KWOK, Linchi; YU, Bei. Spreading social media messages on Facebook: An analysis of restaurant business-to-consumer communications. Cornell Hospitality Quarterly, v. 54, n. 1, p. 84-94, 2013. LANG, Mark; LEMMERER, Andreas. How and why restaurant patrons value locally sourced foods and ingredients. International Journal of Hospitality Management, v. 77, p. 76-88, 2018.

LEE, C.; HALLAK, R.; SARDESHMUKH, S. R. Innovation, entrepreneurship, and restaurant performance: A higher-order structural model. Tourism Management, v. 53, pp. 215-228, $2016 \mathrm{a}$.

LEE, Ji-Eun. Assessment of organizational climate in the restaurant industry. Journal of foodservice business research, v. 20, n. 4, p. 447-463, 2017.

LEE, Ji-Eun; NELSON, Douglas C.; ALMANZA, Barbara A. The impact of individual health inspectors on the results of restaurant sanitation inspections: Empirical evidence. Journal of Hospitality Marketing \& Management, v. 19, n. 4, p. 326-339, 2010. 
LEE, K.; CONKLIN, M.; BORDI, P.; CRANAGE, D. Restaurants' healthy eating initiatives for children increase parents' perceptions of CSR, empowerment, and visit intentions. International Journal of Hospitality Management, v. 59, p. 60-71, $2016 \mathrm{~b}$.

LEE, Kiwon et al. Restaurants' healthy eating initiatives for children increase parents' perceptions of CSR, empowerment, and visit intentions. International Journal of Hospitality Management, v. 59, p. 60-71, 2016.

LEE, Kiwon; LEE, Youngmi; KWON, Sooyoun. How nutrition information frame affects parents' perceptions of restaurants: The moderating role of information credibility. International journal of hospitality management, v. 46, p. 112-119, 2015.

LEE, Sangtak; MCCLEARY, Ken. The relationship between perceived health, health attitude, and healthy offerings for seniors at family restaurants. Cornell Hospitality Quarterly, v. 54, n. 3, p. 262-273, 2013.

LEE, Seoki; HWANG, Johye; HYUN, Martin Yongho. Mobile services as a marketing tool to enhance restaurant revenue: An exploratory study. Journal of Hospitality Marketing \& Management, v. 19, n. 5, p. 464-479, 2010.

LEE, Yee Ming; SOZEN, Erol. Food allergy knowledge and training among restaurant employees. International Journal of Hospitality Management, v. 57, p. 52-59, 2016.

LEE, Yee Ming; XU, Hui. Food allergy knowledge, attitudes, and preparedness among restaurant managerial staff. Journal of Foodservice Business Research, v. 18, n. 5, p. 454-469, 2015.

LEIPER, Neil; STEAR, Lloyd. Tourism-related business failures: the case of The Spice Islands Restaurant. Journal of Vacation Marketing, v. 15, n. 4, p. 367-380, 2009.

LINE, Nathan D. et al. Where everybody knows your name: Homophily in restaurant atmospherics. Journal of Hospitality Marketing \& Management, v. 21, n. 1, p. 1-19, 2012.

LINE, Nathaniel D.; HANKS, Lydia. The other customer: The impact of self-image in restaurant patronage. Journal of Foodservice Business Research, v. 20, n. 3, p. 268-285, 2017.

LINE, Nathaniel D.; HANKS, Lydia; ZHANG, Lu. Sustainability communication: The effect of message construals on consumers' attitudes towards green restaurants. International Journal of Hospitality Management, v. 57, pp. 143-151, 2016.

LIU, Pei; LEE, Yee Ming. An investigation of consumers' perception of food safety in the restaurants. International Journal of Hospitality Management, v. 73, p. 29-35, 2018.

LIU, Pei; LEE, Yee Ming. An investigation of restaurant food safety performance: A comparison between ethnic and nonethnic and chain and independent restaurants in Louisiana. Journal of Foodservice Business Research, v. 20, n. 2, p. 204-217, 2017.

LLOYD, Alison E.; LUK, Sherriff TK. Interaction behaviors leading to comfort in the service encounter. Journal of Services marketing, v. 25, n. 3, p. 176-189, 2011. 
LO, Ada; KING, Brian; MACKENZIE, Murray. Restaurant customers' attitude toward sustainability and nutritional menu labels. Journal of Hospitality Marketing \& Management, v. 26, n. 8, p. 846-867, 2017.

LOUREIRO, M. L.; RAHMANI, D. The incidence of calorie labeling on fast food choices: a comparison between stated preferences and actual choices. Economics \& Human Biology, v. 22, pp. 82-93, 2016.

LU, Lu; GURSOY, Dogan. Does offering an organic food menu help restaurants excel in competition? An examination of diners' decision-making. International Journal of Hospitality Management, v. 63, p. 72-81, 2017.

MA, Jing; GHISELLI, Richard. Measuring, monitoring, and managing the green practices in midsized restaurants in China. Journal of foodservice business research, v. 19, n. 1, p. 64-76, 2016.

MAKKI, Abdullah M.; OZTURK, Ahmet Bulent; SINGH, Dipendra. Role of risk, self-efficacy, and innovativeness on behavioral intentions for mobile payment systems in the restaurant industry. Journal of Foodservice Business Research, v. 19, n. 5, p. 454-473, 2016.

MILLER, Brian. Compensation practices in restaurants and the impact on service quality. Journal of Foodservice Business Research, v. 13, n. 1, p. 24-35, 2010.

MIRANDA, Francisco Javier; RUBIO, Sergio; CHAMORRO, Antonio. The web as a marketing tool in the Spanish foodservice industry: Evaluating the websites of Spain's top restaurants. Journal of Foodservice Business Research, v. 18, n. 2, p. 146-162, 2015.

MOROSAN, Cristian. Customers' adoption of biometric systems in restaurants: An extension of the technology acceptance model. Journal of Hospitality Marketing \& Management, v. 20, n. 6, p. 661-690, 2011.

MOZEIK, Celeste K. et al. The adoption of restaurant-based e-service. Journal of Foodservice Business Research, v. 12, n. 3, p. 247-265, 2009.

MURPHY, K.; OLSEN, M. Dimensions of a high-performance management system. International Journal of Contemporary Hospitality Management, v. 21, Iss 7 pp. 836-853, 2009.

MURPHY, Kevin S. et al. An exploratory case study of factors that impact the turnover intentions and job satisfaction of multi-unit managers in the casual theme segment of the US restaurant industry. Journal of Foodservice Business Research, v. 12, n. 3, p. 200-218, 2009.

MURPHY, Kevin S. et al. Does mandatory food safety training and certification for restaurant employees improve inspection outcomes? International Journal of Hospitality Management, v. 30, n. 1, p. 150-156, 2011.

NAMKUNG, Young; JANG, Soo Cheong Shawn. Effects of restaurant green practices on brand equity formation: do green practices really matter? International Journal of Hospitality Management, v. 33, p. 85-95, 2013. 
NAMKUNG, Young; JANG, Soo Cheong Shawn; CHOI, Soo Keun. Customer complaints in restaurants: Do they differ by service stages and loyalty levels? International Journal of Hospitality Management, v. 30, n. 3, p. 495-502, 2011.

NAMKUNG, Young; JANG, Soo Cheong. Service failures in restaurants: which stage of service failure is the most critical? Cornell Hospitality Quarterly, v. 51, n. 3, p. 323-343, 2010.

NIKBIN, D.; MARIMUTHU, M.; HYUN, S. S. Influence of perceived service fairness on relationship quality and switching intention: An empirical study of restaurant experiences. Current Issues in Tourism, v. 19, n. 10, pp. 1005-1026, 2013.

NOONE, Breffni M.; COULTER, R. Craig. Applying modern robotics technologies to demand prediction and production management in the quick-service restaurant sector. Cornell Hospitality Quarterly, v. 53, n. 2, p. 122-133, 2012.

OKUMUS, Bendegul et al. Psychological factors influencing customers' acceptance of smartphone diet apps when ordering food at restaurants. International Journal of Hospitality Management, v. 72, p. 67-77, 2018.

OZTURK, Ahmet Bulent et al. Understanding the mobile payment technology acceptance based on valence theory: A case of restaurant transactions. International Journal of Contemporary Hospitality Management, v. 29, n. 8, p. 2027-2049, 2017.

PANTELIDIS, Ioannis S. Electronic meal experience: A content analysis of online restaurant comments. Cornell Hospitality Quarterly, v. 51, n. 4, p. 483-491, 2010.

PARIKH, Anish A. et al. Comparative content analysis of professional, semi-professional, and usergenerated restaurant reviews. Journal of foodservice business research, v. 20, n. 5, p. 497-511, 2017.

PARK, Haeik et al. Consumer perceptions and emotions about sanitation conditions in full-service restaurants. Journal of Foodservice Business Research, v. 19, n. 5, p. 474-487, 2016.

PARK, Sang Hee et al. Assessing the provision of nutritional information on quick service restaurant menu item choices for college students. Journal of foodservice business research, v. 16, n. 4, p. 329-346, 2013.

PARSA, H. G. et al. Why restaurants fail? Part II-The impact of affiliation, location, and size on restaurant failures: Results from a survival analysis. Journal of Foodservice Business Research, v. 14, n. 4, p. 360-379, 2011.

PARSA, H. G. et al. Why restaurants fail? Part IV: The relationship between restaurant failures and demographic factors. Cornell Hospitality Quarterly, v. 56, n. 1, p. 80-90, 2015.

PIDD, Ken; ROCHE, Ann; KOSTADINOV, Victoria. Trainee chefs' experiences of alcohol, tobacco and drug use. Journal of Hospitality and Tourism Management, v. 21, p. 108-115, 2014.

POULSTON, Jill; YIU, Albert Yau Kwong. Profit or principles: Why do restaurants serve organic food? International Journal of Hospitality Management, v. 30, n. 1, p. 184-191, 2011. 
POWERS, Monica et al. College students' health attitudes, perceptions of restaurant menu items, and purchase intentions. Journal of foodservice business research, v. 20, n. 4, p. 464-488, 2017.

RHOU, Y.; SINGAL, M.; KOH, Y. CSR and financial performance: The role of CSR awareness in the restaurant industry. International Journal of Hospitality Management, v. 57, pp. 30-39, 2016.

RIVERA, Manuel; SHANI, Amir. Attitudes and orientation toward vegetarian food in the restaurant industry: An operator's perspective. International Journal of Contemporary Hospitality Management, v. 25, n. 7, p. 1049-1065, 2013.

RO, Heejung. Customer dissatisfaction responses to restaurant service failures: Insights into noncomplainers from a relational perspective. Journal of Hospitality Marketing \& Management, v. 24, n. 4, p. 435-456, 2015.

ROBERTS, Kevin R.; BARRETT, Betsy B. Restaurant managers' beliefs about food safety training: An application of the Theory of Planned Behavior. Journal of Foodservice Business Research, v. 14, n. 3, p. 206-225, 2011.

ROEST, Henk; RINDFLEISCH, Aric. The influence of quality cues and typicality cues on restaurant purchase intention. Journal of Retailing and Consumer Services, v. 17, n. 1, p. 10-18, 2010.

RUIZ-MOLINA, María-Eugenia; GIL-SAURA, Irene; BERENGUER-CONTRÍ, Gloria. Information and communication technology as a differentiation tool in restaurants. Journal of Foodservice Business Research, v. 17, n. 5, p. 410-428, 2014.

SALEHI-ESFAHANI, Saba et al. Investigating information adoption tendencies based on restaurants' user-generated content utilizing a modified information adoption model. Journal of Hospitality Marketing \& Management, v. 25, n. 8, p. 925-953, 2016.

SATOW, Yumi E.; INCIARDI, John F.; WALLACE, Sharonda P. Factors used by restaurant customers to predict sanitation levels. Journal of Foodservice Business Research, v. 12, n. 2, p. 170-179, 2009.

SCHAARSCHMIDT, Mario; HÖBER, Björn. Digital booking services: comparing online with phone reservation services. Journal of Services Marketing, v. 31, n. 7, p. 704-719, 2017.

SCHUBERT, Franziska et al. Exploring consumer perceptions of green restaurants in the US. Tourism and Hospitality Research, v. 10, n. 4, p. 286-300, 2010.

SELF, John T.; JONES, Margie Feree; BOTIEFF, Mark. Where restaurants fail: A longitudinal study of micro locations. Journal of Foodservice Business Research, v. 18, n. 4, p. 328-340, 2015.

SEO, Soobin et al. How Have Restaurant Firms Responded to Food Safety Crises? Evidence From Media Coverage. Journal of foodservice business research, v. 21, n. 1, p. 83-105, 2018.

SEO, Soobin; HWANG, Johye. Does gender matter? Examining gender composition's relationships with meal duration and spending in restaurants. International Journal of Hospitality Management, v. 42, p. 61-70, 2014. 
SHAPOVAL, Valeriya; MURPHY, Kevin S.; SEVERT, Denver. Does service quality really matter at Green restaurants for Millennial consumers? The moderating effects of gender between loyalty and satisfaction. Journal of foodservice business research, v. 21, n. 6, p. 591-609, 2018.

SHARMA, Amit; MOON, Joonho; STROHBEHN, Catherine. Restaurant's decision to purchase local foods: Influence of value chain activities. International Journal of Hospitality Management, v. 39, p. 130-143, 2014.

SHARMA, Shilpa et al. Impact of point of selection nutrition information on meal choices at a table-service restaurant. Journal of foodservice business research, v. 14, n. 2, p. 146-161, 2011.

SHIN, Joongwon; MATTILA, Anna S. When Pizza Doesn't Sound as Good as Usual: Restrained Versus Unrestrained Eaters' Responses to Gluten-Free Menu Items. Cornell Hospitality Quarterly, v. 59, n. 4, p. 397-410, 2018.

SHIN, Yeon Ho et al. Consumers' willingness to patronize locally sourced restaurants: The impact of environmental concern, environmental knowledge, and ecological behavior. Journal of Hospitality Marketing \& Management, v. 26, n. 6, p. 644-658, 2017b.

SHIN, Yeon Ho et al. Locally Sourced Restaurant: Consumers Willingness to Pay. Journal of foodservice business research, v. 21, n. 1, p. 68-82, 2017c.

SHIN, Yeon Ho et al. The effect of environmental values and attitudes on consumer willingness to pay more for organic menus: A value-attitude-behavior approach. Journal of Hospitality and Tourism Management, v. 33, p. 113-121, 2017a.

SILBER, Ilan et al. Recovery strategies for service failures: The case of restaurants. Journal of Hospitality Marketing \& Management, v. 18, n. 7, p. 730-740, 2009.

SINGH, Dipendra; KIM, Yen-Soon; FEINSTEIN, Andrew Hale. Internet utilization as a medium for training employees in multi-unit restaurants. Journal of foodservice business research, v. 14, n. 2, p. 122-145, 2011.

STASTNY, Sherri Nordstrom; EVENSON, Alexa; MOZUMDAR, Arupendra. Effect of nutrition facts panel and ingredient declaration on customer satisfaction and nutrition perceptions in a tableservice restaurant at midday meal. Journal of foodservice business research, v. 14, n. 4, p. 310333, 2011.

SUN, Yu-Hua Christine. Menu Nutrition Labels' Effects on Customers' Attitudes toward Menu and Restaurant Dining Intentions-The Moderating Role of Psychosocial Factors. Journal of foodservice business research, v. 16, n. 2, p. 139-154, 2013.

SUSSKIND, Alex M. Communication richness: Why some guest complaints go right to the topAnd others do not. Cornell Hospitality Quarterly, v. 56, n. 3, p. 320-331, 2015

SUSSKIND, Alex M.; CURRY, Benjamin. A Look at How Tabletop Technology Influences Table Turn and Service Labor Usage in Table-Service Restaurants. Cornell Hospitality Quarterly, v. 60, n. 3, p. 233-236, 2018. 
SUSSKIND, Alex; VICCARI, Anthony. A look at the relationship between service failures, guest satisfaction, and repeat-patronage intentions of casual dining guests. Cornell Hospitality Quarterly, v. 52, n. 4, p. 438-444, 2011.

SWEENEY, Jill; ARMSTRONG, Robert W.; JOHNSON, Lester W. The effect of cues on service quality expectations and service selection in a restaurant setting: A retrospective and prospective commentary. Journal of Services Marketing, v. 30, n. 2, p. 136-140, 2016.

TAM, Jackie LM; SHARMA, Piyush; KIM, Namwoon. Attribution of success and failure in intercultural service encounters: the moderating role of personal cultural orientations. Journal of Services Marketing, v. 30, n. 6, p. 643-658, 2016.

TANG, Guiyao et al. The importance of ethical leadership in employees' value congruence and turnover. Cornell Hospitality Quarterly, v. 56, n. 4, p. 397-410, 2015.

TEWS, Michael J.; MICHEL John W.; STAFFORD, Kathryn. Does Fun Pay? The Impact of Workplace Fun on Employee Turnover and Performance. Cornell Hospitality Quarterly, v. 54, n. 4, pp. 370-382, 2013.

TEWS, Michael J.; STAFFORD, Kathryn; TRACEY, J. Bruce. What matters most? The perceived importance of ability and personality for hiring decisions. Cornell Hospitality Quarterly, v. 52, n. 2, p. 94-101, 2011.

THUNSTRÖM, L.; NORDSTRÖM, J.; SHOGREN, J. F.; EHMKE, M.; VELD, K. V. Strategic self-ignorance. Journal of Risk and Uncertainty, v. 52, n. 2, p. 117-136, 2016.

TOTTEN, Jeff W.; MCKAY, Sandra; KONELL, Sid. Another look at consumers' ratings of quickservice restaurant meals. Journal of Foodservice Business Research, v. 12, n. 4, p. 292-316, 2009.

TRACEY, J. Bruce et al. A field study of new employee training programs: industry practices and strategic insights. Cornell Hospitality Quarterly, v. 56, n. 4, p. 345-354, 2015.

VICTORINO, Liana; BOLINGER, Alexander R. Scripting employees: An exploratory analysis of customer perceptions. Cornell Hospitality Quarterly, v. 53, n. 3, p. 196-206, 2012.

VU, Huy Quan et al. Exploring tourist dining preferences based on restaurant reviews. Journal of Travel Research, v. 58, n. 1, p. 149-167, 2017.

WANG, Xi; TANG, Liang Rebecca; KIM, Eojina. More than words: Do emotional content and linguistic style matching matter on restaurant review helpfulness? International Journal of Hospitality Management, v. 77, p. 438-447, 2018.

WANG, Yao-Fen et al. Developing green management standards for restaurants: An application of green supply chain management. International Journal of Hospitality Management, v. 34, p. 263-273, 2013.

WANG, Yao-Fen. Modeling predictors of restaurant employees' green behavior: Comparison of six attitude-behavior models. International Journal of Hospitality Management, v. 58, p. 66-81, 2016. 
WEI, W.; TORRES, E.; HUA, N. Improving consumer commitment through the integration of selfservice technologies: A transcendent consumer experience perspective. International Journal of Hospitality Management, v. 59, p. 105-115, 2016.

WEI, Wei; MIAO, Li. Effects of calorie information disclosure on consumers' food choices at restaurants. International Journal of Hospitality Management, v. 33, p. 106-117, 2013.

WEN, Han; KWON, Junehee. Restaurant servers' risk perceptions and risk communicationrelated behaviors when serving customers with food allergies in the US. International Journal of Hospitality Management, v. 64, p. 11-20, 2017.

WILKINSON, Richard F. Leadership role profile and job satisfaction of restaurant general managers. Journal of Foodservice Business Research, v. 13, n. 4, p. 331-345, 2010.

YANG, Sung-Byung et al. An empirical examination of online restaurant reviews on Yelp. com: A dual coding theory perspective. International Journal of Contemporary Hospitality Management, v. 29, n. 2, p. 817-839, 2017.

YEH, Shih-Shuo; HUAN, Tzung-Cheng. Assessing the impact of work environment factors on employee creative performance of fine-dining restaurants. Tourism Management, v. 58, p. 119-131, 2017.

YEPES, Maryam F. Mobile tablet menus: attractiveness and impact of nutrition labeling formats on millennials' food choices. Cornell Hospitality Quarterly, v. 56, n. 1, p. 58-67, 2015.

YOO, Joanne Jung-Eun; KIM, Taegoo Terry; LEE, Gyehee. When customers complain: The value of customer orientation in service recovery. Cornell Hospitality Quarterly, v. 56, n. 4, p. 411-426, 2015.

YORK, Valerie K. et al. Using the Theory of Planned Behavior to elicit restaurant employee beliefs about food safety: Using surveys versus focus groups. Journal of Foodservice Business Research, v. 12, n. 2, p. 180-197, 2009.

YOUN, Hyewon; GU, Zheng. Predict US restaurant firm failures: The artificial neural network model versus logistic regression model. Tourism and Hospitality Research, v. 10, n. 3, p. 171-187, 2010.

ZHAI, Shixiao et al. Mapping the popularity of urban restaurants using social media data. Applied Geography, v. 63, p. 113-120, 2015.

ZHANG, Lu; HANKS, Lydia. Online reviews: The effect of cosmopolitanism, incidental similarity, and dispersion on consumer attitudes toward ethnic restaurants. International Journal of Hospitality Management, v. 68, p. 115-123, 2018.

ZHANG, Zili et al. Booking now or later: Do online peer reviews matter? International Journal of Hospitality Management, v. 77, p. 147-158, 2018.

ZHANG, Ziqiong et al. The impact of e-word-of-mouth on the online popularity of restaurants: A comparison of consumer reviews and editor reviews. International Journal of Hospitality Management, v. 29, n. 4, p. 694-700, 2010.

ZHENG, X.; GUO, X. E-retailing of restaurant services: pricing strategies in a competing online environment. Journal of the Operational Research Society, v. 67, n. 11, p. 1408-1418, 2016. 